\title{
The Arabidopsis Thylakoid Chloride Channel AtCLCe Functions in Chloride Homeostasis and Regulation of Photosynthetic Electron Transport
}

\author{
Andrei Herdean ${ }^{1}$, Hugues Nziengui ${ }^{1 \dagger}$, Ottó Zsiros ${ }^{2 \dagger}$, Katalin Solymosi ${ }^{3 \dagger}$, Gyözö Garab ${ }^{2}$, \\ Björn Lundin ${ }^{1}$ and Cornelia Spetea ${ }^{1 *}$ \\ ${ }^{1}$ Department of Biological and Environmental Sciences, University of Gothenburg, Gothenburg, Sweden, ${ }^{2}$ Biological \\ Research Center, Hungarian Academy of Sciences, Szeged, Hungary, ${ }^{3}$ Department of Plant Anatomy, Eötvös Loránd \\ University, Budapest, Hungary
}

\section{OPEN ACCESS}

Edited by:

Richard Sayre

New Mexico Consortium at Los Alamos National Labs, USA

Reviewed by:

Peter Jahns,

Heinrich Heine University Duesseldorf,

Germany

Nabil l. Elsheery,

Tanta University, Egypt

*Correspondence:

Cornelia Spetea

cornelia.spetea.wiklund@bioenv.gu.se

${ }^{\dagger}$ These authors have contributed equally to this work.

Specialty section:

This article was submitted to

Plant Physiology,

a section of the journal

Frontiers in Plant Science

Received: 22 October 2015

Accepted: 21 January 2016

Published: 09 February 2016

Citation:

Herdean A, Nziengui $H$, Zsiros $O$,

Solymosi K, Garab G, Lundin B and Spetea C (2016) The Arabidopsis Thylakoid Chloride Channel AtCLCe

Functions in Chloride Homeostasis and Regulation of Photosynthetic Electron Transport.

Front. Plant Sci. 7:115. doi: 10.3389/fpls.2016.00115
Chloride ions can be translocated across cell membranes through $\mathrm{Cl}^{-}$channels or $\mathrm{Cl}^{-} / \mathrm{H}^{+}$exchangers. The thylakoid-located member of the $\mathrm{Cl}^{-}$channel CLC family in Arabidopsis thaliana (AtCLCe) was hypothesized to play a role in photosynthetic regulation based on the initial photosynthetic characterization of clce mutant lines. The reduced nitrate content of Arabidopsis clce mutants suggested a role in regulation of plant nitrate homeostasis. In this study, we aimed to further investigate the role of AtCLCe in the regulation of ion homeostasis and photosynthetic processes in the thylakoid membrane. We report that the size and composition of proton motive force were mildly altered in two independent Arabidopsis clce mutant lines. Most pronounced effects in the clce mutants were observed on the photosynthetic electron transport of dark-adapted plants, based on the altered shape and associated parameters of the polyphasic OJIP kinetics of chlorophyll a fluorescence induction. Other alterations were found in the kinetics of state transition and in the macro-organization of photosystem II supercomplexes, as indicated by circular dichroism measurements. Pre-treatment with $\mathrm{KCl}$ but not with $\mathrm{KNO}_{3}$ restored the wild-type photosynthetic phenotype. Analyses by transmission electron microscopy revealed a bow-like arrangement of the thylakoid network and a large thylakoid-free stromal region in chloroplast sections from the dark-adapted clce plants. Based on these data, we propose that AtCLCe functions in $\mathrm{Cl}^{-}$homeostasis after transition from light to dark, which affects chloroplast ultrastructure and regulation of photosynthetic electron transport.

\footnotetext{
Keywords: Arabidopsis thaliana, CLC channel, chlorophyll fluorescence, electron microscopy, photosynthetic electron transport, proton motive force, state transition, thylakoid membrane
}

\footnotetext{
Abbreviations: $C D$, circular dichroism; Chl, chlorophyll; CLC, chloride channel; Cyt, cytochrome; $\mathrm{F}_{\mathrm{v}} / \mathrm{F}_{\mathrm{m}}$, maximum quantum yield of photosystem II photochemistry; FR, far red; LHC, light harvesting complex; $\Delta \Psi$, membrane potential; $\mathrm{NPQ}$, non-photochemical quenching; P700, primary donor of photosystem I; PC, plastocyanin; PI, performance index; $\Delta \mathrm{pH}$, $\mathrm{pH}$ gradient; $\mathrm{g}_{\mathrm{H}}{ }^{+}$, proton conductivity through ATP synthase; $\nu_{\mathrm{H}}{ }^{+}$, proton flux; PMF, proton motive force; PS, photosystem; $\Phi(\mathrm{II})$, PSII efficiency; ST, state transition; TEM, transmission electron microscopy.
} 


\section{INTRODUCTION}

Photosynthesis is essential for life on Earth. A key element of photosynthesis is conversion of sunlight energy into organic carbon via the generation of a membrane electrochemical potential gradient for protons $\left(\mathrm{H}^{+}\right)$, also known as the proton motive force (PMF). To generate PMF, pigments (chlorophylls and carotenoids) bound to proteins in light harvesting complexes (LHCs) absorb photons and transfer their excitation energy to the reaction centers of photosystems (PS). Here the excitation is converted into charge separation, which drives electron transport from PSII to PSI via the cytochrome $\mathrm{b}_{6} f\left(\mathrm{Cyt}_{6} f\right)$ complex. The net result of this process is the oxidation of water molecules (oxygen evolution) and the reduction of $\mathrm{NADP}^{+}$, which is associated with translocation of $\mathrm{H}^{+}$into the thylakoid lumen. A light-driven cyclic electron transport around PSI and Cyt $\mathrm{b}_{6} f$ is also operative, which does not evolve oxygen, nor induce $\mathrm{NADP}^{+}$reduction but only contributes to $\mathrm{H}^{+}$translocation. It is the water oxidation and photosynthetic electron transportcoupled $\mathrm{H}^{+}$translocation into the lumen that generates PMF, which is composed of transmembrane $\mathrm{H}^{+}$concentration $(\Delta \mathrm{pH})$ and electrical potential $(\Delta \Psi)$ gradients. Both PMF components can drive ATP synthesis, whereas it is thought that only the $\Delta \mathrm{pH}$ component can activate the photoprotective PsbS- and xanthophyll cycle-dependent components of non-photochemical quenching $(N P Q)$ while down-regulating the electron transport during the step of plastoquinol oxidation at Cyt $\mathrm{b}_{6} f$ complex (Kramer et al., 2003). The role of $\Delta \Psi$ in regulation of photosynthesis is less clear, but there is recent evidence in Arabidopsis thaliana (hereafter referred to as Arabidopsis) that ion channels, such as the thylakoid $\mathrm{K}^{+}$channel TPK3, partially dissipate $\Delta \Psi$ to allow more $\mathrm{H}^{+}$to enter the lumen and thus a significant $\Delta \mathrm{pH}$ to be formed, balancing photoprotection and photochemical efficiency (Carraretto et al., 2013).

Potassium, chloride, magnesium, and calcium are the major ions in thylakoids, and changes in their homeostasis are expected to impact membrane architecture, protein conformation, and electron transport rates (Anderson et al., 2012; Pribil et al., 2014; Finazzi et al., 2015; Pottosin and Shabala, 2015). Evidence for fluxes of these ions across the spinach thylakoid membrane accompanying the inward movement of $\mathrm{H}^{+}$during electron transport reactions was provided already by Hind et al. (1974). The proteins involved in $\mathrm{K}^{+}$fluxes have been recently characterized in Arabidopsis (Carraretto et al., 2013; Armbruster et al., 2014; Kunz et al., 2014). Voltage-dependent chloride channel activities in the thylakoid membrane have been reported in Peperomia metallica (Schönknecht et al., 1988) and in the alga Nitellopsis obtusa (Pottosin and Schönknecht, 1995), but thus far the proteins responsible for those activities have not been identified.

In plants, three gene families for $\mathrm{Cl}^{-}$transport have been described thus far, namely slow-anion channels (SLAC), aluminum-activated malate transporters (ALMT), and $\mathrm{Cl}^{-}$ channels (CLCs; Barbier-Brygoo et al., 2011). The Arabidopsis genome codes for seven CLC members (AtCLCa to AtCLCg), localized in various intracellular membrane compartments, and thought to be either $\mathrm{Cl}^{-}$channels or $\mathrm{Cl}^{-} / \mathrm{H}^{+}$exchangers.
Nevertheless, several of them have been also reviewed as nitrate transporters (Krapp et al., 2014). More specifically, AtCLCa and AtCLCb are tonoplast-located $2 \mathrm{NO}_{3}^{-} / 1 \mathrm{H}^{+}$antiporters (De Angeli et al., 2006; von der Fecht-Bartenbach et al., 2010). AtCLCc and AtCLCg are also located in the tonoplast, AtCLCd, and AtCLCf in the Golgi apparatus, and AtCLCe in the thylakoid membrane (Teardo et al., 2005; Marmagne et al., 2007; von der Fecht-Bartenbach et al., 2007; Lv et al., 2009). The selectivity and mechanism of anion transport for these five AtCLCs are unknown (Barbier-Brygoo et al., 2011).

Various physiological functions have been proposed for AtCLCs, based on the phenotypic characterization of corresponding Arabidopsis knockout mutants. AtCLCa, AtCLCb, and AtCLCe are required to maintain normal cellular $\mathrm{NO}_{3}^{-}$levels (De Angeli et al., 2007; von der Fecht-Bartenbach et al., 2010), and in addition AtCLCe may regulate the photosynthetic activity of thylakoids (Marmagne et al., 2007). AtCLCc participates in both $\mathrm{NO}_{3}^{-}$and $\mathrm{Cl}^{-}$homeostasis, and regulates stomatal movement and salt tolerance (Jossier et al., 2010). AtCLCg is also involved in salt tolerance by altering $\mathrm{Cl}^{-}$homeostasis in mesophyll cells (Nguyen et al., 2015). AtCLCd has been proposed to regulate lumenal $\mathrm{pH}$ in the trans-Golgi network (von der Fecht-Bartenbach et al., 2007), and to act as a negative regulator of plant innate immunity (Guo et al., 2014). The physiological function of AtCLCf is still unknown.

In this study, we addressed the question about the physiological role of AtCLCe in the thylakoid membrane. We show that AtCLCe loss-of-function mutation modifies the arrangement of thylakoid network in chloroplasts and alters photosynthetic electron transport following transfer from light to dark.

\section{MATERIALS AND METHODS}

\section{Plant Growth Conditions}

A. thaliana cv. Columbia (Col-0) plants and two clce mutants in the same background were grown in soil for 7-8 weeks in a growth chamber (CLF PlantMaster, Plant Climatics, Wertingen, Germany) using 8-h-light $\left(120 \mu \mathrm{mol}\right.$ photons $\mathrm{m}^{-2}$ $\left.\mathrm{s}^{-1}\right) / 16$-h-dark cycles at $22^{\circ} \mathrm{C}$ and $70 \%$ relative humidity. The SALK_010237 (clce-2) and SALK_21945C (clce-3) mutants were obtained from the Arabidopsis Biological Resource Center (ABRC, https://www.arabidopsis.org/abrc/). The clce-2 line was previously characterized by Marmagne et al. (2007).

\section{RNA Isolation and RT-PCR}

Total RNA was isolated from rosette leaves of 7-8-week old plants using TriZol reagent (Invitrogen), treated with RNasefree DNAse (Thermo Scientific) to prevent DNA contamination and then purified using HiBind RNA mini columns (Omega Bio-Tek) according to the manufacturer's instructions. cDNA was synthesized using $1 \mu \mathrm{g}$ of total RNA through iScript cDNA synthesis Kit (Bio-Rad). Finally, $2 \mu \mathrm{L}$ of reverse transcription reaction were used as template to amplify AtCLCe and $\beta$ ATPase cDNA fragments using Dream Taq DNA Polymerase Kit (Thermo Scientific). The following primers were used for 
the AtCLCe (At4g35440): forward TCCAAGTGTTGAAATTGG AGC and reverse AGGTGTAACAGTCCATGGCAC, and for mitochondrial ATP synthase $\beta$-subunit (At5g08680) selected as reference gene: forward GATCATGACATCTCTCGAGG and reverse TGGTAAGGAGCAAGGAGATC.

\section{Determination of Leaf Chlorophyll (Chl) Content}

Chl content was determined from leaf discs after extraction in $96 \%(v / v)$ ethanol at $65^{\circ} \mathrm{C}$ for $10 \mathrm{~min}$ followed by spectrophotometry (Lichtenthaler and Wellburn, 1983). The Chl content was expressed per leaf area and per fresh weight.

\section{Electrochromic Band Shift (ECS) Measurements}

ECS measurements were carried out using a Pulse Amplitude Modulated Chl fluorometer (Dual PAM-100, Walz, Effeltrich, Germany) equipped with a P515/535 module (Schreiber and Klughammer, 2008). Leaves of $30 \mathrm{~min}$ dark-adapted plants were illuminated with actinic red light at 100 or $650 \mu \mathrm{mol}$ photons $\mathrm{m}^{-2} \mathrm{~s}^{-1}$ for 2 , 5, or $10 \mathrm{~min}$. After each illumination period, the light was switched off and the dark interval relaxation kinetics (DIRK) of the ECS signal were recorded for $60 \mathrm{~s}$ according to Cruz et al. (2001) to estimate PMF size $\left(\mathrm{ECS}_{\mathrm{t}}\right)$ and relative contribution of $\Delta \mathrm{pH}$ and $\Delta \Psi$ to PMF. Before each PMF measurement, a saturating single turnover 5- $\mu$ s flash of $200,000 \mu \mathrm{mol}$ photons $\mathrm{m}^{-2} \mathrm{~s}^{-1}$ was applied to determine $\mathrm{ECS}_{\mathrm{ST}}$, which was used to normalize ECS .

For determination of $\mathrm{H}^{+}$conductivity of the thylakoid membrane mainly through ATP synthase $\left(\mathrm{gH}^{+}\right)$, the leaves were exposed to light at $650 \mu \mathrm{mol}$ photons $\mathrm{m}^{-2} \mathrm{~s}^{-1}$ for $10 \mathrm{~min}$. At specific time points, the light was switched off to record the ECS signal decay during 600-ms dark intervals. The $\mathrm{g}_{\mathrm{H}}{ }^{+}$parameter was calculated as $1 /$ time constant for decay derived from single exponential fittings of the ECS decay during the first $100 \mathrm{~ms}$ (Cruz et al., 2005). The steady state proton flux $\left(v_{\mathrm{H}}{ }^{+}\right)$was calculated as $\mathrm{g}_{\mathrm{H}}{ }^{+}{ }^{*} \mathrm{ECS}_{\mathrm{t}} / \mathrm{ECS}_{\mathrm{ST}}$ (Cruz et al., 2005).

\section{Kinetics of Chl a Fluorescence Induction}

Fast Chl $a$ fluorescence induction (OJIP) kinetics were recorded using a Plant Efficiency Analyser (Handy-PEA, Hansatech, King's Lynn, Norfolk, UK) by applying saturating red actinic light $\left(635 \mathrm{~nm}, 3500 \mu \mathrm{mol}\right.$ photons $\left.\mathrm{m}^{-2} \mathrm{~s}^{-1}, 1 \mathrm{~s}\right)$ on plants during dark adaptation intervals of 1-15 min. Where indicated, recorded data points were double normalized to minimum $\left(F_{0}\right)$ and maximum $\left(F_{m}\right)$ fluorescence. The time to reach $F_{m}$ ( $\mathrm{t}_{\mathrm{Fm}}$ in $\left.\mathrm{ms}\right)$, the maximum quantum yield of PSII $\left(F_{v} / F_{m}\right)$, the performance index $(P I)$, variable fluorescence yield at $J$ step $\left(V_{J}\right)$, variable fluorescence yield at $I$ step $\left(V_{I}\right)$, and the turnover number of $\mathrm{Q}_{\mathrm{A}}$ $(N)$ were calculated using Hansatech PEA Plus v1.10 software according to Strasser et al. (2004). Where indicated, detached leaves were incubated in $150 \mathrm{mM} \mathrm{KCl}$ or $\mathrm{KNO}_{3}$ for $30 \mathrm{~min}$ in growth light $\left(120 \mu \mathrm{mol}\right.$ photons $\left.\mathrm{m}^{-2} \mathrm{~s}^{-1}\right)$ followed by adaptation in darkness for $15 \mathrm{~min}$ before the OJIP measurements.

Slow Chl $a$ fluorescence induction and recovery kinetics were recorded using the Dual PAM-100 instrument (Walz) on 30-min dark-adapted plants using red actinic light of 100 or $650 \mu \mathrm{mol}$ photons $\mathrm{m}^{-2} \mathrm{~s}^{-1}$ for $10 \mathrm{~min}$ followed by $5 \mathrm{~min}$ in darkness. The non-photochemical quenching $(N P Q)$ and quantum yield of PSII $[\Phi(I I)]$ were calculated using the following equations: $\Phi(I I)=\left(F_{m}{ }^{\prime}-F\right) / F_{m}{ }^{\prime}$, where $F_{m}{ }^{\prime}$ is defined as the fluorescence value at the plateau level reached during application of a saturating pulse, and $F$ is defined as the fluorescence level during illumination averaged for $0.2 \mathrm{~s}$ before applying the saturating pulse; $N P Q=\left(F_{m}-F_{m}{ }^{\prime}\right) / F_{m}{ }^{\prime}$, where $F_{m}$ is the fluorescence value at the plateau level reached during application of a saturating pulse on the $30 \mathrm{~min}$ dark-adapted leaf before the onset of illumination.

\section{P700 Oxidation-Reduction Kinetics}

To monitor the oxidation-reduction kinetics of PSI, absorbance changes at $830 \mathrm{~nm}$ (reflecting the redox state of P700, i.e., PSI primary donor) were recorded using the Dual PAM-100 (Walz) instrument. The 830-nm transmittance was subtracted from the simultaneous recording at $875 \mathrm{~nm}$, and calibrated according to the Dual PAM-100 built-in routine, and finally displayed as P700 $\Delta \mathrm{I} / \mathrm{I}^{*} 10^{3}$. Before the measurement, plants were adapted to light $\left(120 \mu \mathrm{mol}\right.$ photons $\left.\mathrm{m}^{-2} \mathrm{~s}^{-1}, 1 \mathrm{~h}\right)$. Oxidation-reduction kinetics were recorded by applying saturating red actinic light pulses (635 $\mathrm{nm}, 20,000 \mu \mathrm{mol}$ photons $\mathrm{m}^{-2} \mathrm{~s}^{-1}, 200 \mathrm{~ms}$ ) together with far red (FR) light $(730 \mathrm{~nm})$ during the illumination period and after 1, 2, 3, 4, and 5 min of dark adaptation. Half of each darkadaptation interval ( $30 \mathrm{~s}$ ) was done in the presence of FR light to fully oxidize P700 before applying the saturation pulse.

\section{State Transition (ST) Kinetics}

ST measurements were carried out according to Lunde et al. (2000) on $30 \mathrm{~min}$ dark-adapted plants using the Dual PAM100 (Walz) instrument. For determination of $F_{m}$ values in the dark and in either state 1 or state 2 , a saturating pulse of red actinic light $\left(5000 \mu \mathrm{mol}\right.$ photons $\mathrm{m}^{-2} \mathrm{~s}^{-1}, 800 \mathrm{~ms}$ ) was applied. To induce state 2, leaves were illuminated for $15 \mathrm{~min}$ with red actinic light $\left(100 \mu \mathrm{mol}\right.$ photons $\mathrm{m}^{-2} \mathrm{~s}^{-1}$; "state 2 light"). For transition to state 1 , leaves were exposed for $15 \mathrm{~min}$ to red light supplemented with FR light (“state 1 light"). The $q T$ parameter was calculated as $\left(F_{m 2}-F_{m 3}\right) / F_{m 2}$, where $F_{m 2}$ is the fluorescence value at the plateau level reached during application of the saturating pulse after $15 \mathrm{~min}$ illumination with "state 1 light" and $F_{m 3}$ after 15 min illumination with "state 2 light." The $q S$ parameter was calculated as $\left[\left(F_{I^{\prime}}-F_{I}\right)-\left(F_{I I^{\prime}}-F_{I I}\right)\right] /\left(F_{I^{\prime}}-F_{I}\right)$ according to Damkjaer et al. (2009), where $F_{I}$ and $F_{I I}$ are the steady-state fluorescence levels in the presence of FR light in state 1 and state 2, respectively, and $F_{I^{\prime}}$ and $F_{I I^{\prime}}$ are the steady-state fluorescence levels in the absence of FR light in state 1 and state 2 , respectively.

\section{Circular Dichroism (CD) Spectroscopy}

CD measurements were carried out on a J-815 spectropolarimeter (JASCO, Tokyo, Japan). Detached, waterinfiltrated leaves were placed between two glass slides in an optical cell. Spectra were recorded at room temperature between

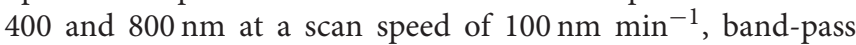
of $3 \mathrm{~nm}$ and step size of $1 \mathrm{~nm}$. For each sample, 3-4 scans 
were averaged. Spectra were normalized to the absorption of the red-most peak of the spectra recorded at the same time as the CD spectra and were corrected for baseline distortions. Measurements were repeated on three different leaves for each genotype. Amplitudes of psi-type CD bands, at around (+)505, $(-) 675$, and $(+) 690 \mathrm{~nm}$, were determined using the reference wavelengths of 550,600, and $750 \mathrm{~nm}$, respectively.

\section{Transmission Electron Microscopy (TEM)}

Sections $(\sim 2 \times 2 \mathrm{~mm})$ cut from the central parts of leaf blades of 7 -week-old plants were fixed in $2.5 \%(v / v)$ glutaraldehyde for 4 days, then post-fixed in $1 \% \mathrm{OsO}_{4}(w / v)$ for $2 \mathrm{~h}$. Fixatives were buffered with $70 \mathrm{mM} \mathrm{Na} 2 \mathrm{HPO}_{4}-\mathrm{KH}_{2} \mathrm{PO}_{4}$ (pH 7.2). After fixation, samples were rinsed in the same buffer. After dehydration in an alcohol series, samples were embedded in Durcupan ACM resin (Fluka, Buchs, Switzerland). Ultrathin sections (thickness $70 \mathrm{~nm}$ ) were cut with a Reichert Jung Ultracut E microtome (Reichert-Jung AG, Vienna, Austria), mounted on copper grids and contrasted with 5\% uranyl acetate and Reynolds' lead citrate solution. The sections were visualized with a Hitachi 7100 TEM microscope at $75 \mathrm{kV}$ accelerating voltage.

ImageJ software was used to measure granum diameter (at the middle of perpendicular granum sections) on the micrographs. Calculations were done on 210-350 randomly chosen grana originating from 35 different chloroplasts taken randomly from 35 different mesophyll cells per treatment.

\section{Statistical Analyses}

Each mean is given \pm SEM for at least 5 plants. The data sets were compared between genotypes by one-way ANOVA using OriginPro 8 for Windows. Significant differences were considered at $P<0.05$.

\section{RESULTS}

\section{Phenotype of clce Mutants}

To investigate the role of AtCLCe in the thylakoid membrane, we have characterized the phenotype of two Arabidopsis T-DNA insertion lines in Col-0 background. The clce-2 mutant was initially characterized by Marmagne et al. (2007). We have identified an additional T-DNA insertion line (clce-3) in the same background (Figure 1A). Both genotypes lacked the AtCLCe transcript, as revealed by RT-PCR analyses (Figure 1B). Neither line displayed obvious growth differences from wildtype (Figure 1C) and no statistically significant differences in Chl content, Chl $a / b$ ratio or specific weight of leaves were obtained (Table 1).

Next we compared the photosynthetic performance of darkadapted plants based on $\mathrm{Chl} a$ fluorescence induction parameters. The $F_{v} / F_{m}$ parameter used as an indicator of the maximum quantum yield of PSII was found slightly but significantly lower in $15 \mathrm{~min}$ dark-adapted clce mutants than in wildtype due to a significantly lower $F_{m}$ (Table 1). Another Chl fluorescence parameter used as indicator of the overall plant vitality, is the performance index $(P I)$ of dark-adapted plants (Strasser et al., 2000). PI was found significantly reduced in the clce mutants, suggesting that they experience stress during dark-adaptation. These data indicate that AtCLCe loss-of-function mutation lowers photosynthetic performance in dark-adapted plants.

\section{Proton Motive Force and Proton Flux}

To determine if PMF partitioning into $\Delta \mathrm{pH}$ and $\Delta \Psi$ has been altered in the clce mutants as compared to wildtype, leaves were illuminated for $10 \mathrm{~min}$ at either 100 or $650 \mu \mathrm{mol}$ photons $\mathrm{m}^{-2}$ $\mathrm{s}^{-1}$ and the DIRK of ECS were recorded according to Cruz et al. (2001). In the mutants, a mild but significant increase in $\Delta \Psi$ was observed at both light intensities, and consequently a decrease in $\Delta \mathrm{pH}$ occurred (Figure 2 and Supplementary Figure 1A). This suggests a minor alteration by the AtCLCe loss-of-function in the ion distribution across the thylakoid membrane during illumination. It is of note that differences in PMF partitioning were also observed at $5 \mathrm{~min}$ but not after shorter illumination at $650 \mu \mathrm{mol}$ photons $\mathrm{m}^{-2} \mathrm{~s}^{-1}$ (Supplementary Figure 1B).

Next we investigated the effect of AtCLCe loss-of-function mutation on ATP synthase activity by measuring the thylakoid membrane total PMF $\left(\mathrm{ECS}_{\mathrm{t}}\right)$, conductivity to $\mathrm{H}^{+}\left(\mathrm{g}_{\mathrm{H}}{ }^{+}\right)$, and the $\mathrm{H}^{+}$flux through ATP synthase $\left(v_{\mathrm{H}}{ }^{+}\right)$during exposure for $10 \mathrm{~min}$ to $650 \mu \mathrm{mol}$ photons $\mathrm{m}^{-2} \mathrm{~s}^{-1}$. Both mutant lines displayed a mild but significant increase in all three parameters

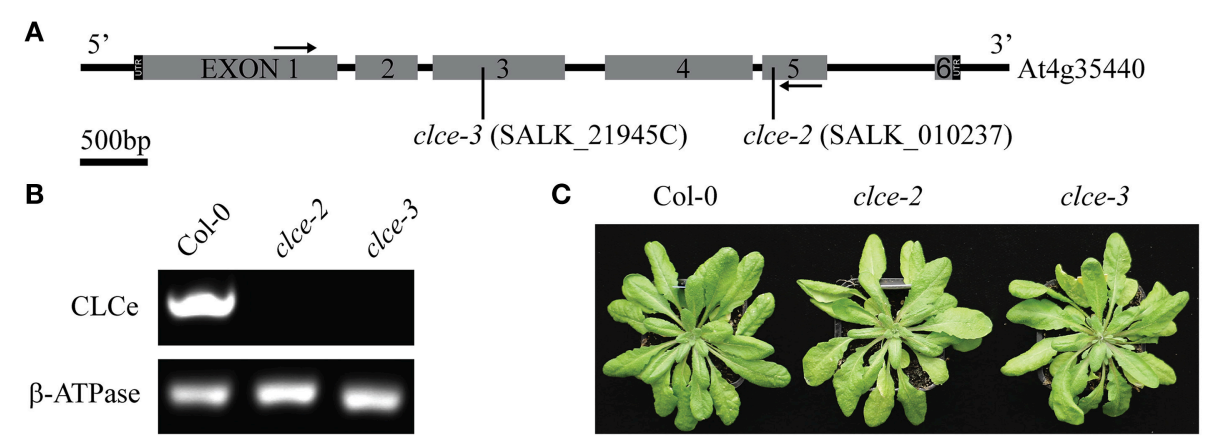

FIGURE 1 | Genotyping of clce mutants used in this study. (A) AtCLCe gene structure with indicated location of T-DNA insert in each of the two clce lines. Black arrows indicate location of primers used for RT-PCR. (B) RT-PCR of total RNA from wild-type (Col-0) and clce leaves with primers specific for the AtCLCe gene and primers for a reference gene as the positive control. (C) Representative photos of 7-week-old plants. 
TABLE 1 | Chlorophyll (Chl) content and photosynthetic performance of dark-adapted plants.

\begin{tabular}{|c|c|c|c|}
\hline Genotype & Col-0 & clce-2 & clce-3 \\
\hline $\mathrm{mg} \mathrm{Chl} \mathrm{cm}{ }^{-2}$ & $0.024 \pm 0.001$ & $0.022 \pm 0.001$ & $0.022 \pm 0.001$ \\
\hline $\mathrm{mg} \mathrm{Chl} \mathrm{g}^{-1}$ fresh weight & $1.335 \pm 0.075$ & $1.232 \pm 0.073$ & $1.248 \pm 0.043$ \\
\hline $\mathrm{mg}$ fresh weight $\mathrm{cm}^{-2}$ & $18.100 \pm 0.693$ & $17.670 \pm 0.403$ & $17.490 \pm 0.429$ \\
\hline Chl a/b & $4.077 \pm 0.042$ & $4.065 \pm 0.051$ & $4.027 \pm 0.029$ \\
\hline$F_{0}$ & $486 \pm 5$ & $500 \pm 10$ & $518 \pm 6$ \\
\hline$F_{m}$ & $2624 \pm 21$ & $2442 \pm 2^{*}$ & $2504 \pm 18^{*}$ \\
\hline$F_{V} / F_{m}$ & $0.814 \pm 0.001$ & $0.795 \pm 0.001^{*}$ & $0.795 \pm 0.001^{*}$ \\
\hline$P l$ & $2.503 \pm 0.044$ & $2.037 \pm 0.042^{*}$ & $2.105 \pm 0.036^{*}$ \\
\hline$V_{J}$ & $0.368 \pm 0.002$ & $0.341 \pm 0.002^{*}$ & $0.337 \pm 0.001^{*}$ \\
\hline$V_{l}$ & $0.750 \pm 0.007$ & $0.598 \pm 0.003^{\star}$ & $0.610 \pm 0.007^{*}$ \\
\hline$t_{F m}(\mathrm{~ms})$ & $338 \pm 43$ & $600 \pm 49^{*}$ & $580 \pm 43^{*}$ \\
\hline$N$ & $59.493 \pm 1.742$ & $127.314 \pm 1.633^{*}$ & $122.545 \pm 3.991^{\star}$ \\
\hline
\end{tabular}

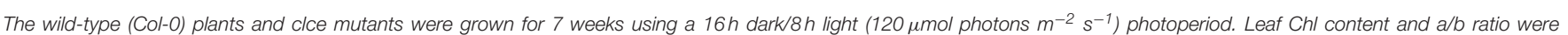

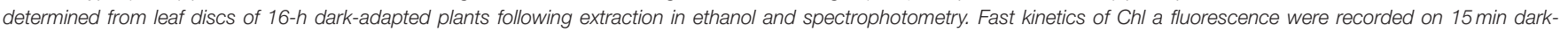

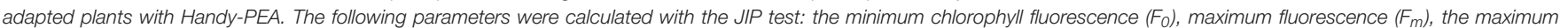
quantum yield $\left(F_{V} / F_{m}\right)$, the performance index $(P I)$, the relative variable fluorescence yield at $2 m s\left(N_{J}\right)$ and at $30 m s\left(V_{I}\right)$, the time to reach $F_{m}\left(t_{F m}\right)$, and the turnover number of $Q_{A}(N)$.

The data are means \pm SEM ( $n=5$ plants). Asterisks indicate significant difference in the studied parameters between Col-0 and the clce mutants (ANOVA, $P<0.05)$.

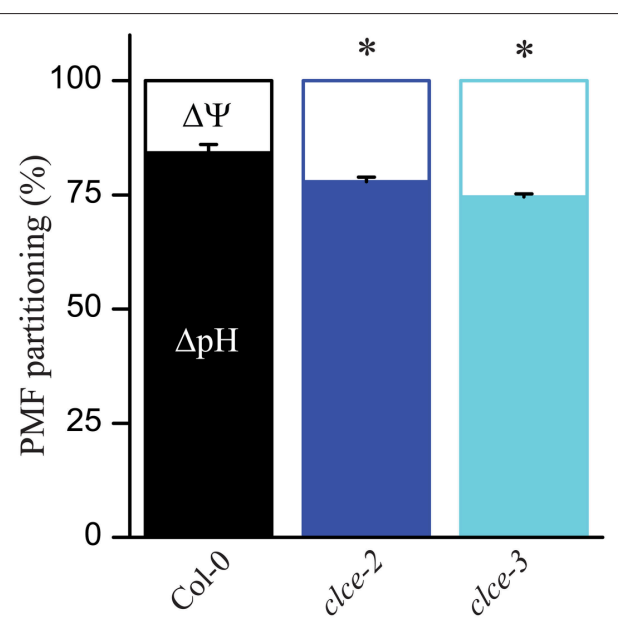

FIGURE 2 | Composition of the proton motive force (PMF). Wild-type and mutant plants were dark-adapted for $30 \mathrm{~min}$ and then illuminated for $10 \mathrm{~min}$ with $650 \mu \mathrm{mol}$ photons $\mathrm{m}^{-2} \mathrm{~s}^{-1}$. Following illumination, kinetics of dark relaxation of the electrochromic shift (ECS) signal were recorded and deconvoluted to determine membrane $\mathrm{pH}$ gradient $(\Delta \mathrm{pH})$ and membrane potential $(\Delta \Psi)$ components. The plotted data are means \pm SEM $(n=8-9$ plants). Asterisks indicate statistically significant differences according to ANOVA $(P<0.05)$.

throughout the illumination time (Figures 3A-C), suggesting enhanced thylakoid membrane $\mathrm{H}^{+}$conductivity. Since the $\mathrm{H}^{+}$ flux is known to linearly correlate with the rate of linear electron flow (Takizawa et al., 2008), the absence of AtCLCe appeared to have a beneficial effect on electron transfer rate in thylakoids during illumination.

\section{Photoprotection and PSII Efficiency}

The $\Delta \mathrm{pH}$ component of PMF regulates PSII efficiency [ $\Phi(I I)]$ and NPQ induction with respect to its fast energy-dependent and slow zeaxanthin-dependent quenching components (Nilkens et al., 2010; Ruban et al., 2012). Based on the lower contribution of $\triangle \mathrm{pH}$ to PMF (Figure 2), we expected a higher $\Phi(I I)$ and lower $N P Q$ in the mutants as compared to wildtype. Instead, we observed a significantly higher steady-state NPQ level in clce after about $10 \mathrm{~min}$ of illumination at 650 and $100 \mu \mathrm{mol}$ photons $\mathrm{m}^{-2}$ $\mathrm{s}^{-1}$ (Figures 4A,B). The $\Phi(I I)$ parameter did not significantly differ between wildtype and mutants during the same set of measurements (Figures 4C,D). NPQ relaxation during the subsequent dark phase after either 650 or $100 \mu \mathrm{mol}$ photons $\mathrm{m}^{-2} \mathrm{~s}^{-1}$ was significantly slower, whereas $\Phi(I I)$ was consistently lower without reaching wild-type levels (Figures 4A-D). The observed effects of AtCLCe loss-of-function mutation on NPQ during illumination may have other cause than the altered relative contribution of $\Delta \mathrm{pH}$ to the PMF. The lower $\Phi(I I)$ after transition from light to dark is in line with the lower $F_{v} / F_{m}$ and PI of dark-adapted plants (Table 1).

\section{Fast Chl Fluorescence Induction and P700 Oxidation-Reduction Kinetics}

The fast kinetics of Chl $a$ fluorescence induction (OJIP) display a polyphasic shape and provide information about the electron transport reactions in the thylakoid membrane (Strasser et al., 2000). The shape of the recorded OJIP kinetics was found altered in 15-min dark-adapted clce mutants (Figure 5A), confirming previous observations (Marmagne et al., 2007). The mutants displayed lower $F_{v} / F_{m}$ values due to lower fluorescence level at the $P$ step $\left(F_{m}\right.$, Table 1$)$. The differences in fluorescence levels appeared already at the $J$ step and become greater at the $I$ step (Figure 5A), corresponding to $V_{J}$ and $V_{I}$ parameters (Table 1). The curves were double normalized to $J$ and $P$, and the curve difference $\left(\Delta \mathrm{F}_{\mathrm{JP}}\right)$ between wildtype and clce mutants was plotted (Figure 5A inset). The resulting peak corresponds to the $I$ step, which has been associated with the redox state of the donor and acceptor side of PSI (Schansker et al., 2005). 


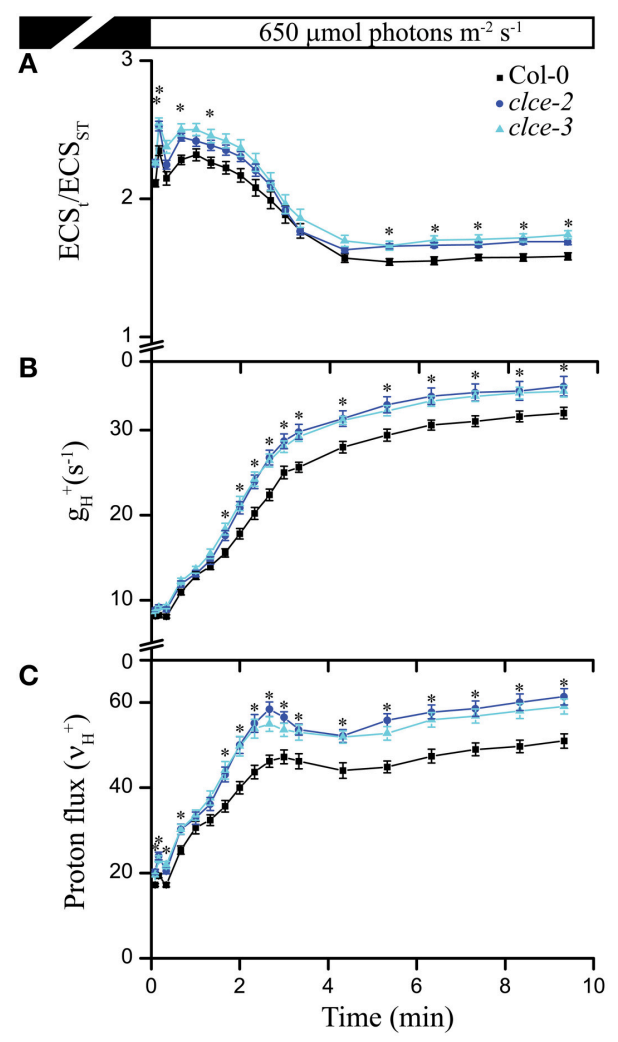

FIGURE 3 | Proton motive force, $\mathrm{H}^{+}$conductivity through ATP synthase, and $\mathbf{H}^{+}$flux. Electrochromic shift measurements (ECS) were performed on 30 min dark-adapted wild-type and mutant plants. (A) Total proton motive force $\left(\mathrm{ECS}_{\mathrm{t}}\right.$ normalized to $\mathrm{ECS}_{\mathrm{ST}}$ ) induction kinetics during illumination at the given light intensity. (B) ATP synthase conductivity $\left(\mathrm{g}_{\mathrm{H}^{+}}{ }^{+}\right.$, and $\mathbf{( C )}$ proton flux $\left(\nu_{\mathrm{H}^{+}}{ }^{+}\right)$were calculated from ECS decay kinetics as described in Section Materials and Methods. The plotted data are means \pm SEM ( $n=8-9$ plants). Asterisks indicate statistically significant differences according to ANOVA $(P<0.05)$.

Marmagne et al. (2007) proposed that the alteration of the $I$ peak is due to the disappearance of a rate limiting step in electron transport between the acceptor side of PSII and of PSI. We also found that the time to reach $F_{m}\left(t_{F m}\right)$ was twice longer in the clce mutants than in wildtype (Table 1). The $N$ parameter for the number of turnovers of PSII primary electron acceptor $\left(\mathrm{Q}_{\mathrm{A}}\right)$, i.e., number of times $\mathrm{Q}_{\mathrm{A}}$ has been reduced in the interval between 0 and $t_{F m}$, was twice as high in the mutants (Table 1). This is in line with the lower fluorescence at the $J$ step, which is also related to $\mathrm{Q}_{\mathrm{A}}$ reduction (Strasser et al., 2000).

Interestingly, pre-treatment of leaves with $150 \mathrm{mM} \mathrm{KCl}$ in the light followed by $15 \mathrm{~min}$ dark-adaptation resulted in similar OJIP kinetics in wildtype and mutants (Figure 5B and inset). Moreover, pre-treatment with $\mathrm{KNO}_{3}$ did not rescue the phenotype observed in the clce mutants (Figure 5C). Instead, the treatment induced a shoulder after the $I$ peak (Figure 5C inset) of unclear origin. The restoration of OJIP wild-type shape in the clce mutants by $\mathrm{KCl}$ treatment suggests that the untreated samples had altered kinetics due to a disturbed $\mathrm{Cl}^{-}$distribution across thylakoids in dark-adapted plants.
Next we investigated if the formation of the $I$ step in wildtype depends on the duration of dark adaptation interval preceding recording. Figure 6A shows the complete absence of the $I$ peak after 1 min of dark adaptation with only the $J-P$ peaks visible. After $2 \mathrm{~min}$ in darkness, the $I$ step started to become visible, and after $5 \mathrm{~min}$ the complete OJIP kinetics could be observed (Figure 6A). The plotted $\Delta \mathrm{F}_{\mathrm{JP}}$ curve differences relative to 15min dark adaptation (Figure 6A inset) indicate that $5 \mathrm{~min}$ in darkness is enough for complete formation of the $I$ step in wildtype. The clce-2 did not develop the $I$ step after either 1 min or longer dark adaptation intervals (Figure 6B and inset). Pre-treatment with $\mathrm{KCl}$ delayed the appearance of the $I$ step from 2 (Figure 6A) to $5 \mathrm{~min}$ in wildtype (Figure 6C), without reaching complete formation of the kinetics within the first 5 min (Figure 6C inset) relative to the $15 \mathrm{~min}$ dark-adapted leaves. These data suggest that formation of the $I$ peak requires at least $5 \mathrm{~min}$ of dark adaptation in wildtype, possibly to allow for re-arrangement of electron transport components between PSII and PSI. KCl treatment appears to delay the formation of the $I$ peak, since it possibly alters $\mathrm{Cl}^{-}$distribution across thylakoids influencing electron transport.

To test if PSI electron transfer was affected in the clce-2 mutant, we used the same dark adaptation intervals as in Figure 6, and recorded P700 oxidation-reduction kinetics (Figure 7A). The reduction of $\mathrm{P} 700^{+}$was found more pronounced in the clce mutant as compared to wildtype after 3 min or longer period in darkness (Figure 7B). This suggests an accelerated electron transfer at PSI, which strengthens the possibility that this caused the lower fluorescence at the $I$ step in Figure 6A. Taken together, the OJIP and P700 kinetics data indicate that in dark-adapted plants CLCe activity is important for $\mathrm{Cl}^{-}$homeostasis of chloroplasts, which in turn influences optimal electron transport between PSII and PSI.

\section{State Transition Kinetics}

We further investigated the consequences of AtCLCe loss-offunction on the plant ability to adapt to changes in light quality, and distribution of excitation energy between PSII and PSI (i.e., state transition) (Tikkanen et al., 2006). Steadystate Chl fluorescence levels of clce-2 in "state 2 light" were found almost identical to the levels recorded in "state 1 light" (Figure 8A), whereas wild-type plants had a significant difference in fluorescence levels before the change of light from state 2 to state 1 . These differences were no longer visible after treatment with $\mathrm{KCl}$ (Figure 8B). During illumination with "state 1 light," steady-state fluorescence levels reached the same values in wildtype and clce-2 mutant, however $\mathrm{F}_{\mathrm{m}}\left(\mathrm{F}_{\mathrm{m} 2}\right)$ was significantly higher in $c l c e-2$, indicating a relatively larger PSII antenna size under state 1 . Nevertheless, the state transition parameter $q T$, indicating the extent of changes in PSII antenna size, was not found significantly different between wildtype $(0.116 \pm 0.011)$ and clce-2 $(0.108 \pm 0.003)$ or clce-3 $(0.114 \pm 0.007)$. We additionally analyzed the kinetics for the transition from state 1 to state 2 (Figures 8A,B insets), which is known to result in decreased PSII antennae size (Bellafiore et al., 2005). The halftime $\left(t_{1 / 2}\right)$ of PSII antenna detachment from PSII during S1 to S2 transition was found significantly lower in the mutant even after 

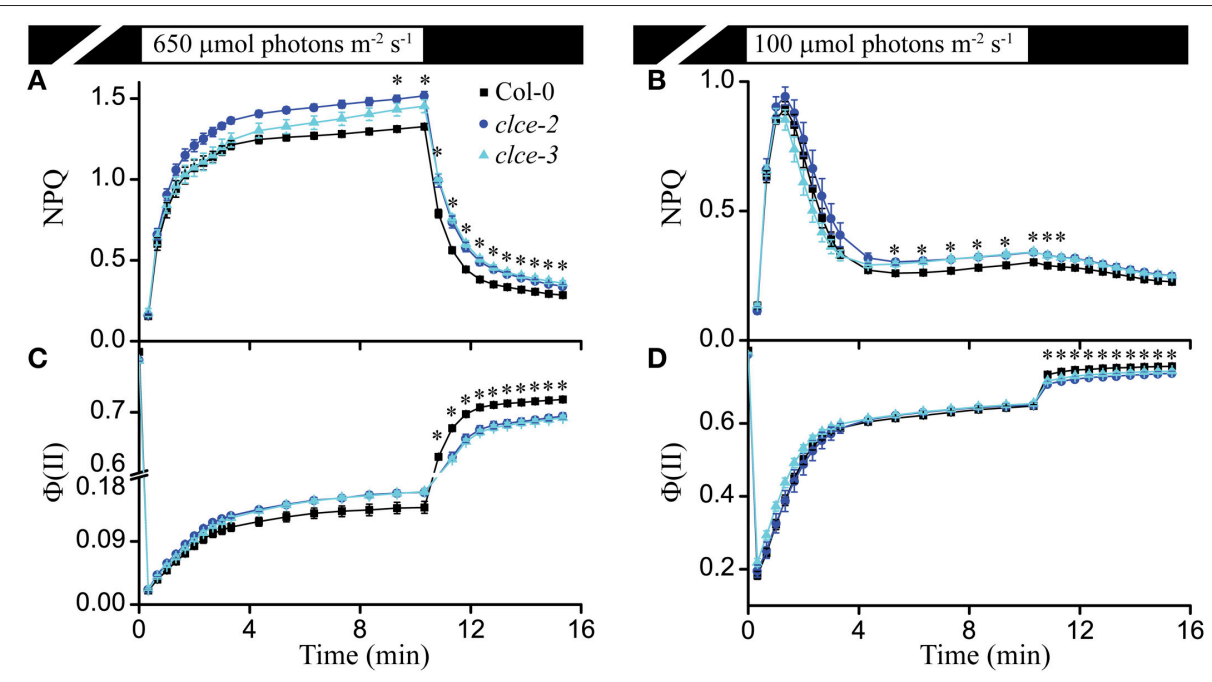

FIGURE 4 | Non-photochemical quenching (NPQ) and PSIl efficiency [ $\mathbf{\Phi ( I I ) ] . ~ N P Q ~ i n d u c t i o n ~ k i n e t i c s ~ o f ~ w i l d - t y p e ~ a n d ~ c l c e ~ p l a n t s ~ w e r e ~ r e c o r d e d ~ d u r i n g ~}$ illumination for $10 \mathrm{~min}$ at $650 \mu \mathrm{mol}$ photons $\mathrm{m}^{-2} \mathrm{~s}^{-1}$ (A) or $100 \mu \mathrm{mol}$ photons $\mathrm{m}^{-2} \mathrm{~s}^{-1}$ (B) followed by 5 min relaxation in darkness. $\Phi$ (II) from the same set of measurements is plotted in (C,D). The plotted data are means \pm SEM ( $n=9$ plants). Asterisks indicate statistically significant differences according to ANOVA $(P<0.05)$.

pre-treatment of the leaves with $\mathrm{KCl}$ (Figure 8C). Surprisingly, untreated wildtype leaves displayed the same $\mathrm{t}_{1 / 2}$ as $\mathrm{KCl}$-treated clce-2. Plant ability to adjust electron transfer to changes in light quality $(q S)$ was found $20 \%$ higher in clce-2 relative to wildtype in untreated leaves, whereas after treatment with $\mathrm{KCl}$ no significant difference was found between mutant and wildtype leaves, but remained 20\% higher than untreated wildtype (Figure 8D).

\section{Circular Dichroism Analysis}

We recorded CD spectra as a non-invasive method of studying the macro-organization of complexes in the thylakoid membrane (Garab and van Amerongen, 2009). In general, both wildtype and the clce-2 mutant displayed typical CD spectra, suggesting no major differences in macro-organization of the complexes. Relatively lower values in the +red psi-type (PSI) and +blue PSI $\mathrm{CD}$ (Figure 9) were observed in the clce-2 mutant as compared to wildtype, indicating a minor perturbation in the organization of PSII-LHCII macrodomains and/or a smaller domain-size compared to wildtype (Garab et al., 1991; Barzda et al., 1994).

\section{Chloroplast Ultrastructure}

Representative TEM images (Figure 10) show that in leaves from the 16-h dark-adapted wild-type plants, chloroplasts were halflens shaped with higher convexity than the elongated and flat chloroplasts observed in the 3-h light-adapted wild-type and clce plants. Dark-adapted clce plants had a more round shape and peculiar ultrastructural features: often a large thylakoid-free stromal zone was located next to the cell wall, and thylakoids with a bow-like arrangement were situated at the vacuolar side of the chloroplast (Figure 10). These features were prominent for $\sim 75 \%$ of clce chloroplast sections, while they were also observed on $\sim 50 \%$ of the wild-type chloroplast sections in the dark-adapted samples. However, in the latter the thylakoids had much less distorted, and less typical bow-like appearance, and the thylakoid-free stroma region was also smaller. The chloroplasts of 3-h light-adapted plants showed regular ultrastructure: their thylakoids were flat and the thylakoid network was arranged parallel to the cell wall, and only small thylakoid-free stroma regions were observed in $\sim 30$ and $10 \%$ of clce and wild-type chloroplast sections, respectively (Figure 10).

Detailed analyses of granum structure revealed no statistically significant differences between clce and wild-type plants either in the dark- or light-adapted states. The average granum diameters were around 450 and $400 \mathrm{~nm}$ in the dark- and in the light-adapted states, respectively. Similarly, the average number of appressed thylakoids per grana (i.e., granum height) was seven in both genotypes, and irrespectively from the light conditions.

There were no apparent differences in the starch contents of clce and wild-type chloroplasts in the light. However, assimilatory starch produced in the light almost completely disappeared during the dark-adaptation period in the clce mutant since only $50 \%$ of the analyzed chloroplast sections contained one small and very thin starch grain. Among the analyzed wild-type chloroplast sections from dark-adapted plants, 95\% contained larger and often several starch grains. Taken together, the AtCLCe loss-offunction mutation influences the chloroplast ultrastructure in dark- but not in light-adapted plants.

\section{DISCUSSION}

\section{Role of AtCLCe in Partial Depolarization of the Thylakoid Membrane}

$\mathrm{Cl}^{-}$has been long considered to be the major counteranion during electron transport-coupled $\mathrm{H}^{+}$translocation, whose import into the lumen is expected to result in rapid 


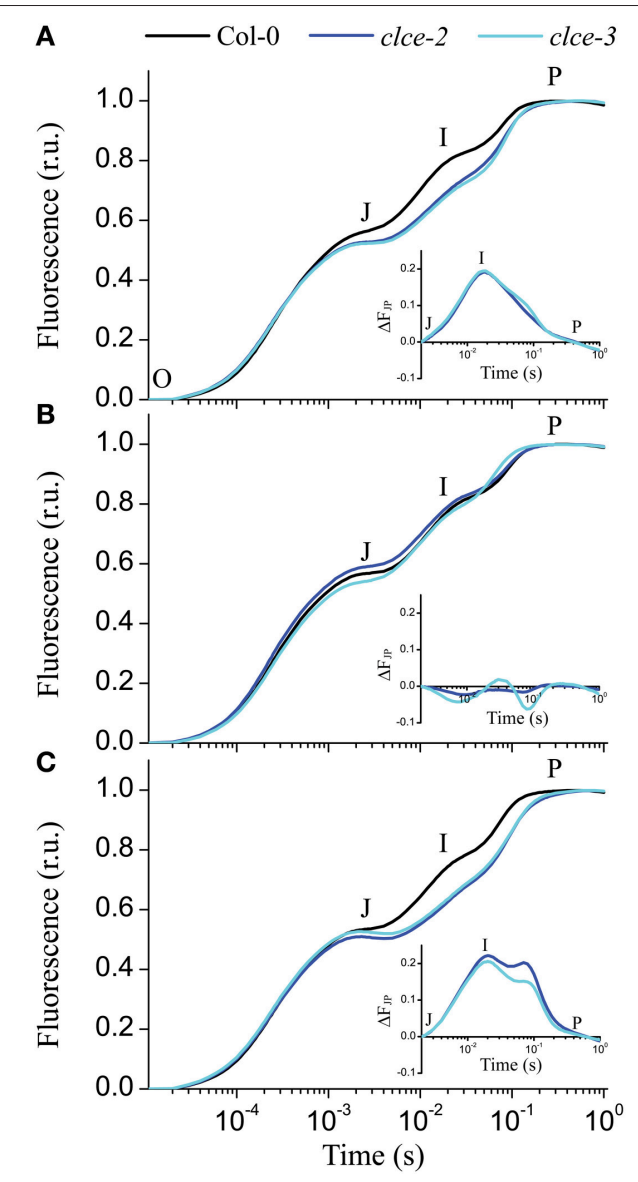

FIGURE 5 | Fast chlorophyll a fluorescence OJIP transients of untreated and salt-treated leaves. Detached wild-type and mutant leaves that have been incubated in the light for $30 \mathrm{~min}$ in water (A), $150 \mathrm{mM} \mathrm{KCl}(\mathbf{B})$, or $150 \mathrm{mM} \mathrm{KNO}_{3}$ (C) were removed from the solution and were dark adapted for $15 \mathrm{~min}$ before OJIP transients were recorded. The transients were double normalized to $F_{0}$ and $F_{m}$. The time points for the calculation of the OJIP parameters are marked: $\mathrm{O}$-the fluorescence intensity at $50 \mu \mathrm{s}, \mathrm{J}-$ at $2 \mathrm{~ms}$, $I$ - at $30 \mathrm{~ms}$, and $P$-the maximum fluorescence intensity. Insets in each plot show the curve difference of double normalized data at $J$ and $P\left(\Delta \mathrm{F}_{\mathrm{JP}}\right)$ between wildtype and each of the clce mutants. The plotted data are means \pm $\operatorname{SEM}(n=5$ plants).

partial depolarization of the thylakoid membrane (Hind et al., 1974). $\mathrm{Cl}^{-}$is thought to be imported in the thylakoid lumen immediately after onset of illumination, and exported to the chloroplast stroma during transitions to dark (Hind et al., 1974). Malfunction of either the import or the export mechanism would result in altered $\mathrm{Cl}^{-}$distribution within the chloroplast, hence altered $\Delta \Psi$ during steady-state photosynthesis. As the only anion channel so far localized to thylakoids, AtCLCe has been hypothesized to be responsible for the partial depolarization of the thylakoid membrane in the light (Finazzi et al., 2015; Pottosin and Dobrovinskaya, 2015). Our data show small but significant increase in $\Delta \Psi$ and total PMF in the clce mutants that occurred only at longer illumination time ( $\geq 5 \mathrm{~min}$, Figures 2, 3A and Supplementary Figure 1). This supports the hypothesized role of AtCLCe in the partial

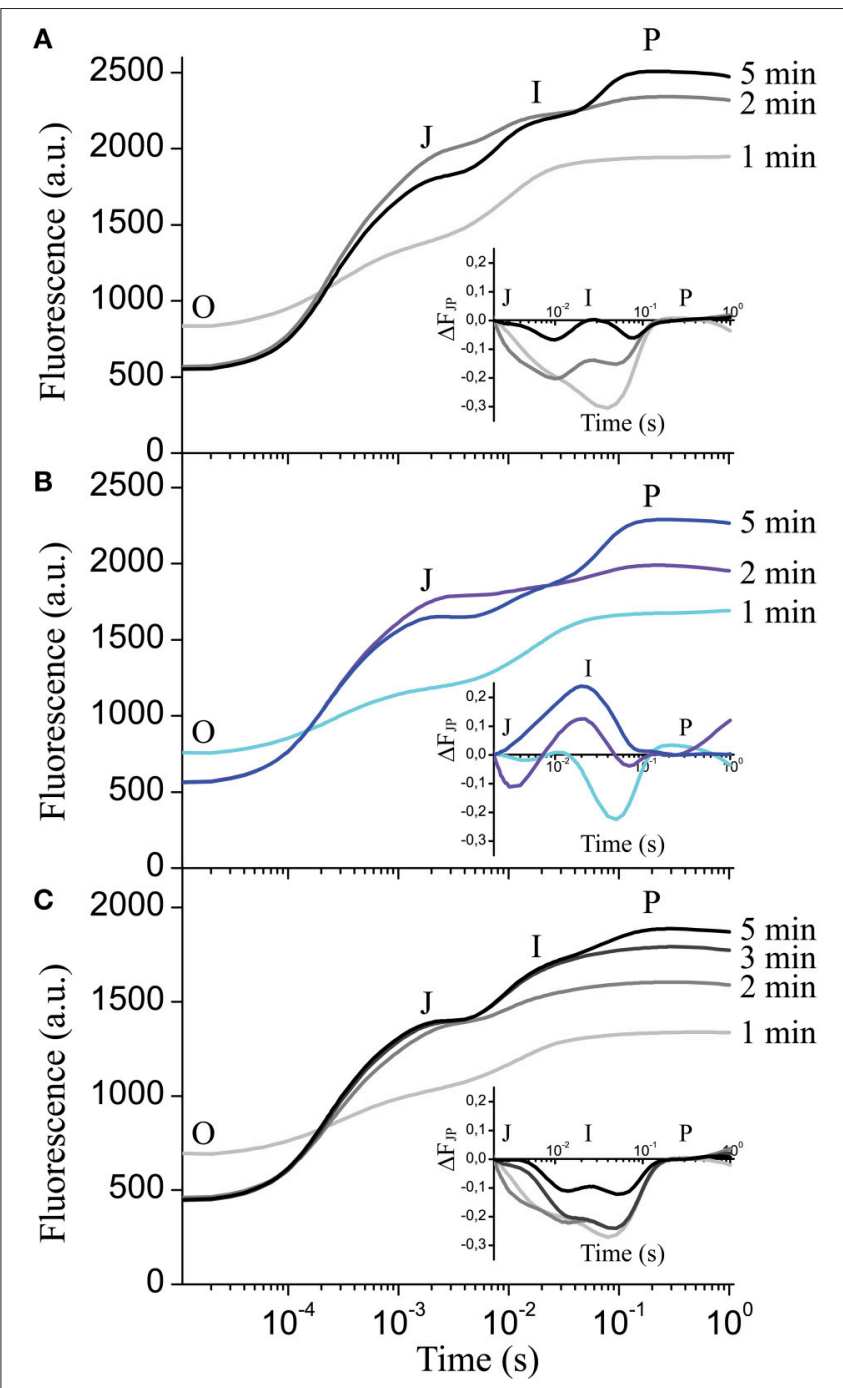

FIGURE 6 | Effect of dark adaptation interval on the fast chlorophyll a fluorescence OJIP transients. OJIP transients were recorded on leaves of wild-type (A) and mutant plants (B), following the indicated dark adaptation intervals. Insets show the curve difference $\left(\Delta F_{J P}\right)$ of double normalized data at $J(2 \mathrm{~ms})$ and $P$, between 15 min dark adapted wildtype and 1, 2, and 5 min dark adapted wildtype (A) or clce-2 (B). (C) OJIP transients were recorded on wild-type leaves pre-incubated with $150 \mathrm{mM} \mathrm{KCl}$ in the light followed by the indicated dark adaptation intervals. Inset shows the curve difference $\left(\Delta F_{J P}\right)$ of double normalized data at $J$ and $\mathrm{P}$ between 15-min dark-adapted $\mathrm{KCl}$-treated wildtype and 1, 2, 3, and 5-min dark-adapted $\mathrm{KCl}$-treated wildtype. The plotted transients are means \pm SEM ( $n=5$ plants).

depolarization of thylakoids, and in addition suggests that activation of AtCLCe may require factors dependent on the light exposure time. $\mathrm{H}^{+}$conductivity through ATP synthase $\left(\mathrm{g}_{\mathrm{H}}{ }^{+}\right)$and proton flux $\left(v_{\mathrm{H}}{ }^{+}\right)$during illumination were also slightly increased (Figures 3B,C). Our data are in agreement with those of Kramer et al. (2003), who proposed that ATP synthase activity is driven by the amplitude of total PMF. Since the effects of the AtCLCe loss-of-function mutation on membrane depolarization and ATP synthase activity are only minor, additional, yet unidentified anion channels must 


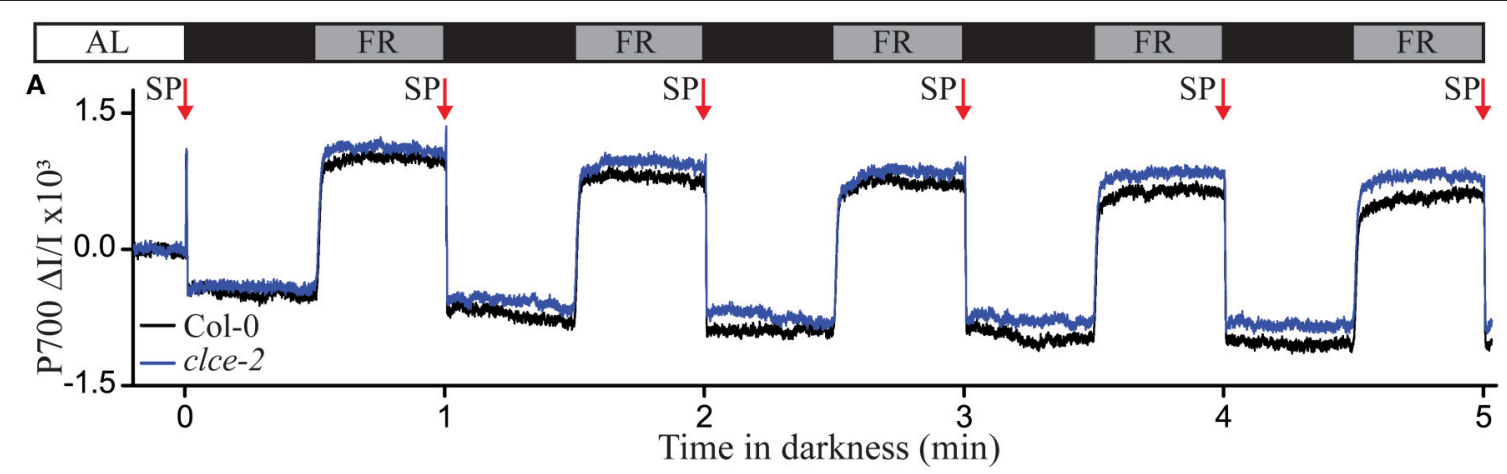

B

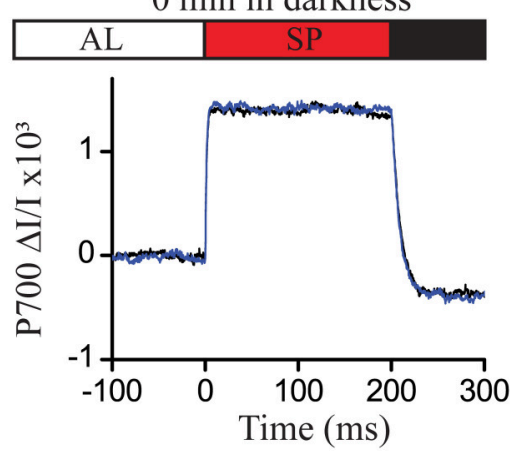

3 min in darkness

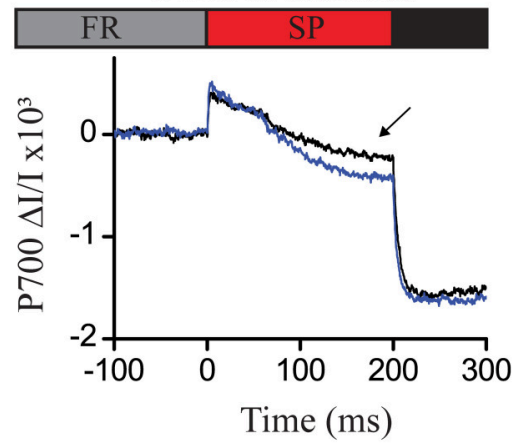

$1 \mathrm{~min}$ in darkness

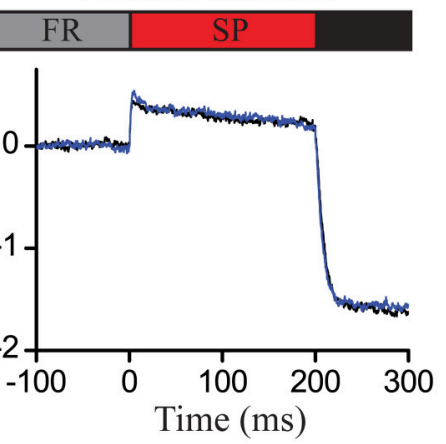

4 min in darkness

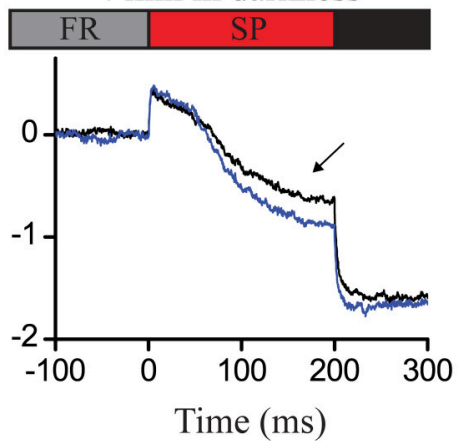

2 min in darkness

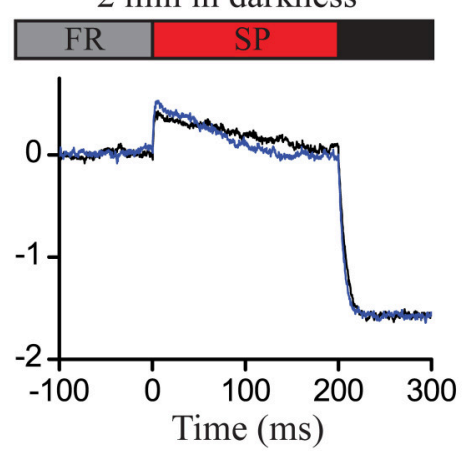

5 min in darkness

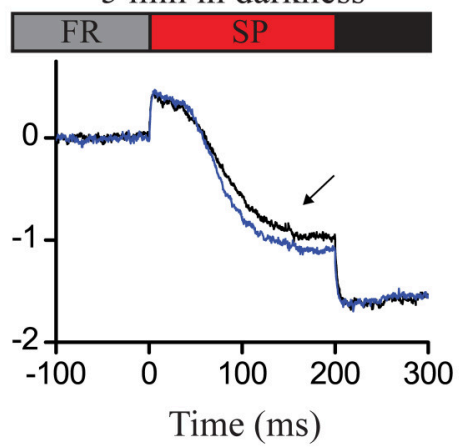

FIGURE 7 | P700 oxidation-reduction kinetics following different dark adaptation intervals. Wild-type and mutant plants have been illuminated with actinic light (AL) of $120 \mu \mathrm{mol}$ photons $\mathrm{m}^{-2} \mathrm{~s}^{-1}$ for $1 \mathrm{~h}$ before recording the P700 signal as difference in transmittance at $875 \mathrm{~nm}$ and $830 \mathrm{~nm}$. The steady state P700 signal in the light was recorded for $15 \mathrm{~s}$, followed by 5 cycles of $30 \mathrm{~s}$ (indicating the P700 reduced state) and $30 \mathrm{~s}$ of far-red (FR) illumination in darkness (indicating the P700

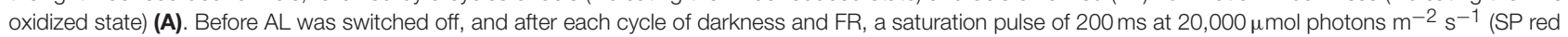
arrow) was applied to record the P700 reduction kinetics (B). The curves were normalized to the initial P700 values in light (A,B -0 min in darkness) or after the FR illumination (B). Black arrows indicate differences in P700 reduction kinetics after 3, 4, and 5 min in darkness. The plotted data are means of measurements from 5 plants.

contribute to the ion compensation of $\mathrm{H}^{+}$uptake into the lumen during illumination.

Our data showing that AtCLCe can regulate $\Delta \Psi$ suggest an electrogenic transport activity, i.e., the protein could work either as a channel or as an exchanger of anion and $\mathrm{H}^{+}$at a stoichiometry of at least $2: 1$, which is the experimentally determined ratio for the algal CLC (Feng et al., 2010). If AtCLCe worked as an anion $/ \mathrm{H}^{+}$exchanger, then it was expected to play an active role in mediating $\mathrm{pH}$ homeostasis or to act as a $\mathrm{H}^{+}$leak to reduce lumenal $\mathrm{pH}$. The mechanism of transport is unknown as well as whether there is coordination between the ATP synthase and AtCLCe to generate the required transmembrane $\mathrm{pH}$ gradient. Such coordination might be indirect via the same protein regulator (e.g., Batelli et al., 2007).

\section{Role of AtCLCe in Regulation of PSII Efficiency and Photoprotection}

$\mathrm{Cl}^{-}$ions are thought to be important for channeling $\mathrm{H}^{+}$ from the oxygen-evolving complex of PSII to the thylakoid lumen (Guskov et al., 2009; Umena et al., 2011). Additionally, 


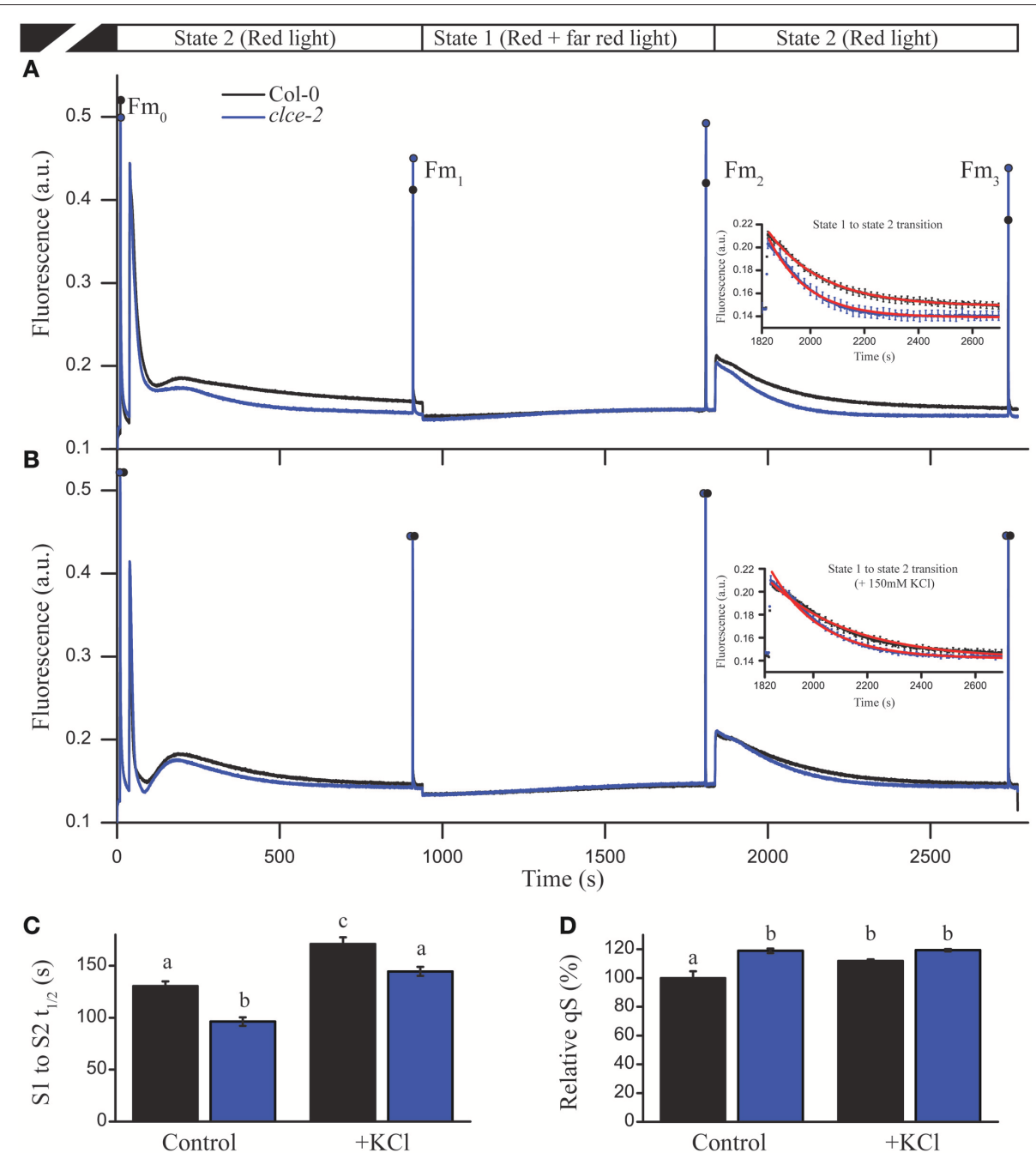

FIGURE 8 | State transition and light harvesting antenna changes in leaves. State transition measurements were performed in untreated (A) and KCl-treated (B) wild-type and mutant leaves. Insets represent state 1 to state 2 transition kinetics. (C) The halftime $\left(\mathrm{t}_{1 / 2}\right)$ of fluorescence relaxation from $\mathrm{S} 1$ to $\mathrm{S} 2 \mathrm{was}$ calculated by fitting an exponential decay function on the fluorescence signal shown in insets. (D) Relative values of qS parameter indicate the ability of the chloroplast to adjust electron transfer to changes in light quality. The plotted data are means \pm SEM ( $n=4-5$ plants). Different letters in panels (C,D) indicate statistically significant differences (ANOVA, $P<0.05)$.

in vitro experiments in media depleted of $\mathrm{Cl}^{-}$showed that PSII particles harbor unstable oxygen-evolving complex (Nash et al., 1985). Under our experimental conditions, PSII efficiency of the clce mutants in the light $[\Phi(I I)]$ was found unchanged, and only during the subsequent dark recovery phase was lower relative to wildtype (Figures $4 \mathrm{C}, \mathbf{D}$ ). These observations suggest that AtCLCe loss-of-function mutation affects PSII activity during dark adaptation rather than during illumination. The cause behind the observed changes in PSII activity could be the thylakoid ultrastructural changes in dark-adapted plants discussed below. Moreover, the lower $F_{v} / F_{m}$ and PI parameters in the clce mutants (Table 1), further indicate an unfavorable organization of the electron transport components in darkness, since these parameters can only be determined correctly after subsequent dark adaptation. The higher steady-state NPQ in clce (Figures 4A,B) cannot be easily explained by the lower $\triangle \mathrm{pH}$ contribution to PMF partitioning (Figure 2), however, other unknown factors related to the $\Delta \Psi$ component may play a role.

\section{Role of AtCLCe in Regulation of Electron Transport via Re-arrangement of Thylakoid Network}

Fast chlorophyll fluorescence $(O J I P)$ kinetics is a useful tool to obtain information about functioning of the electron transport chain. The observed decrease in fluorescence levels at the $I$ step seen in the OJIP kinetics of clce mutants (Figure 5A, Table 1) together with the more pronounced $\mathrm{P} 700^{+}$reduction (Figure 7) indicate accelerated electron transfer at PSI (Schansker et al., 


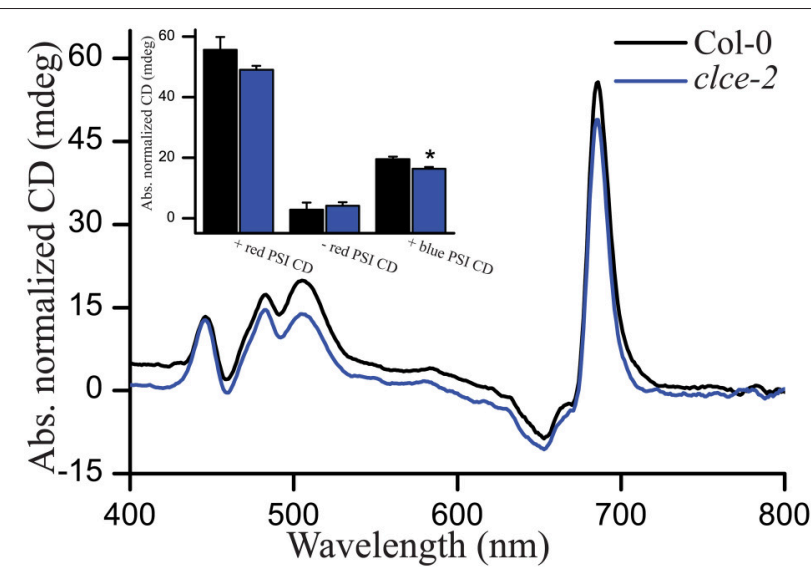

FIGURE 9 | Representative circular dichroism (CD) spectra of $\mathbf{H}_{\mathbf{2}} \mathbf{O}$-infiltrated detached leaves. Insets are amplitudes of psi-type $C D$ bands at around (+)505 nm (+blue), (-)675 nm (-red). and (+)690 nm (+red), with reference wavelengths at 550,600 . and $750 \mathrm{~nm}$, respectively. Data are the means \pm SEM ( $n=3$ plants). Asterisks indicate significant differences (ANOVA, $P<0.05$ ).

2005). The extended time to reach $F_{m}$ suggests that it takes twice as long in the mutants as compared to wildtype to completely close all PSII centers (Figure 5A inset). Moreover, the reduced variable fluorescence at the $J\left(V_{J}\right)$ and $I\left(V_{I}\right)$ steps, as well as the double number of $\mathrm{Q}_{\mathrm{A}}$ reduction events $(N)$ (Table 1) in the clce mutants further suggest an accelerated electron transfer between PSII and PSI as compared to wildtype.

The chloroplast thylakoid lumen undergoes swelling during illumination and shrinkage in darkness (Kirchhoff et al., 2011; Yoshioka-Nishimura et al., 2014). This is thought to be driven mainly by $\mathrm{Cl}^{-}$influx into the thylakoid lumen in the light and efflux in darkness (Kirchhoff, 2013). Expansion of the lumen is necessary for efficient electron transport between Cyt $\mathrm{b}_{6} f$ and PSI via the soluble protein plastocyanin (PC), whose diffusion was proposed to depend on physical space. Additionally, lumen swelling is important for PSII repair (Kirchhoff et al., 2011) and state transition (Chuartzman et al., 2008). Based on this knowledge and our data, we hypothesized that the clce mutants may retain partially swollen thylakoids even after dark adaptation. A swollen lumen would allow for increased mobility of PC, facilitating electron transport from Cyt $\mathrm{b}_{6} f$ to PSI (Kirchhoff et al., 2011). However, no differences in shrinkage/swelling of the thylakoid lumen or significant alterations in granum height and diameter could be resolved by TEM between clce and wild-type plants (Figure 10). The grana and stroma thylakoid network of wildtype and mutant chloroplasts was flat and arranged parallel to the cell walls to maximize photosynthetic energy capture under relatively low light conditions $\left(120 \mu \mathrm{mol}\right.$ photons $\mathrm{m}^{-2}$ $\mathrm{s}^{-1}$ ). Nevertheless, dark-adapted clce chloroplasts had a more pronounced, special bow-like arrangement of the thylakoid network. Similar arrangement of the thylakoid system was reported for the chloroplasts of other photosynthetic species under different stress conditions, including excess of heavy metals that cause disturbances in the ion homeostasis of chloroplasts (e.g., reviewed by Solymosi and Bertrand, 2012). We suggest that the peculiar chloroplast shape and thylakoid arrangement in the clce mutant in darkness could be a mechanism to overcome altered $\mathrm{Cl}^{-}$homeostasis within the chloroplast. The disturbances in chloroplast ultrastructure may in turn alter the functioning of the photosynthetic electrontransport chain. The clce mutant can adapt to light similarly to wildtype as indicated by similar thylakoid ultrastructural changes. This suggests that additional $\mathrm{Cl}^{-}$transport mechanism must exist in thylakoids in the light. This unknown mechanism may be also involved in the restoration of wildtype OJIP kinetics by $\mathrm{KCl}$ pre-treatment (Figure 5B). Similar treatment with $\mathrm{KNO}_{3}$ did not restore the wild-type kinetics (Figure 5C), suggesting that either $\mathrm{NO}_{3}^{-}$homeostasis was not altered in the clce mutant or that changes in $\mathrm{NO}_{3}^{-}$homeostasis did not affect photosynthesis in the clce mutants.

The altered OJIP kinetics observed in clce leaves could also be reproduced in wildtype following dark adaptation for $1 \mathrm{~min}$ (Figures 6A,B). More specifically, this short dark adaptation resulted in a complete absence of the $I$ step in both wildtype and mutant, and only the OJP kinetics were visible. In leaves treated with $\mathrm{KCl}$, we observed a slower formation of the $I$ step, since it became visible only after $5 \mathrm{~min}$ (Figure $6 \mathrm{C}$ and inset), and even after $15 \mathrm{~min}$ the amplitude was still lower relative to untreated leaves (Figures 5A,B). The observation of a delay in formation of the $I$ step upon $\mathrm{KCl}$ treatment suggests that ion homeostasis in darkness is important for a proper re-arrangement of the electron transport chain components. We also found a faster transition of clce-2 from state 1 to state 2 (Figures $\mathbf{8 A - C}$ ) as well as the higher $q S$ values (Figure 8D). A possible explanation is that $\mathrm{Cl}^{-}$ ions may directly influence the electrostatic interactions of LHCII with PSII or PSI, which in turn affect their ability to migrate, also leading to an unfavorable arrangement in darkness. The macroorganization of complexes and the structural stability of the chiral macro-domains have been shown to depend on the ionic strength of the medium (Garab et al., 1991; Cseh et al., 2000). Alternatively, a long-lived membrane potential in darkness due to sustained $\mathrm{Cl}^{-}$ions trapped in the thylakoid lumen may also have a negative effect on the dark relaxation of the electron transport chain.

To conclude, our findings suggest that AtCLCe functions in $\mathrm{Cl}^{-}$homeostasis within the chloroplast leading to rearrangement of the electron transport chain in thylakoids after transition from light to dark. Changed $\mathrm{Cl}^{-}$distribution across thylakoids may be one of the strategies to ensure maximum quantum yields and balance photochemical utilization with photoprotection by NPQ upon light-to-dark and dark-to-light transitions. We propose a minor role for AtCLCe in light-driven $\mathrm{Cl}^{-}$import into the thylakoid lumen, and a major role in $\mathrm{Cl}^{-}$ export to the chloroplast stroma upon dark adaptation. The major $\mathrm{Cl}^{-}$import mechanism driven by membrane potential produced during illumination remains to be identified. $\mathrm{Cl}^{-}$ export by AtCLCe in darkness would hence be driven by the inversed membrane potential across the thylakoid membrane, which occurs when light is switched off, and would facilitate 


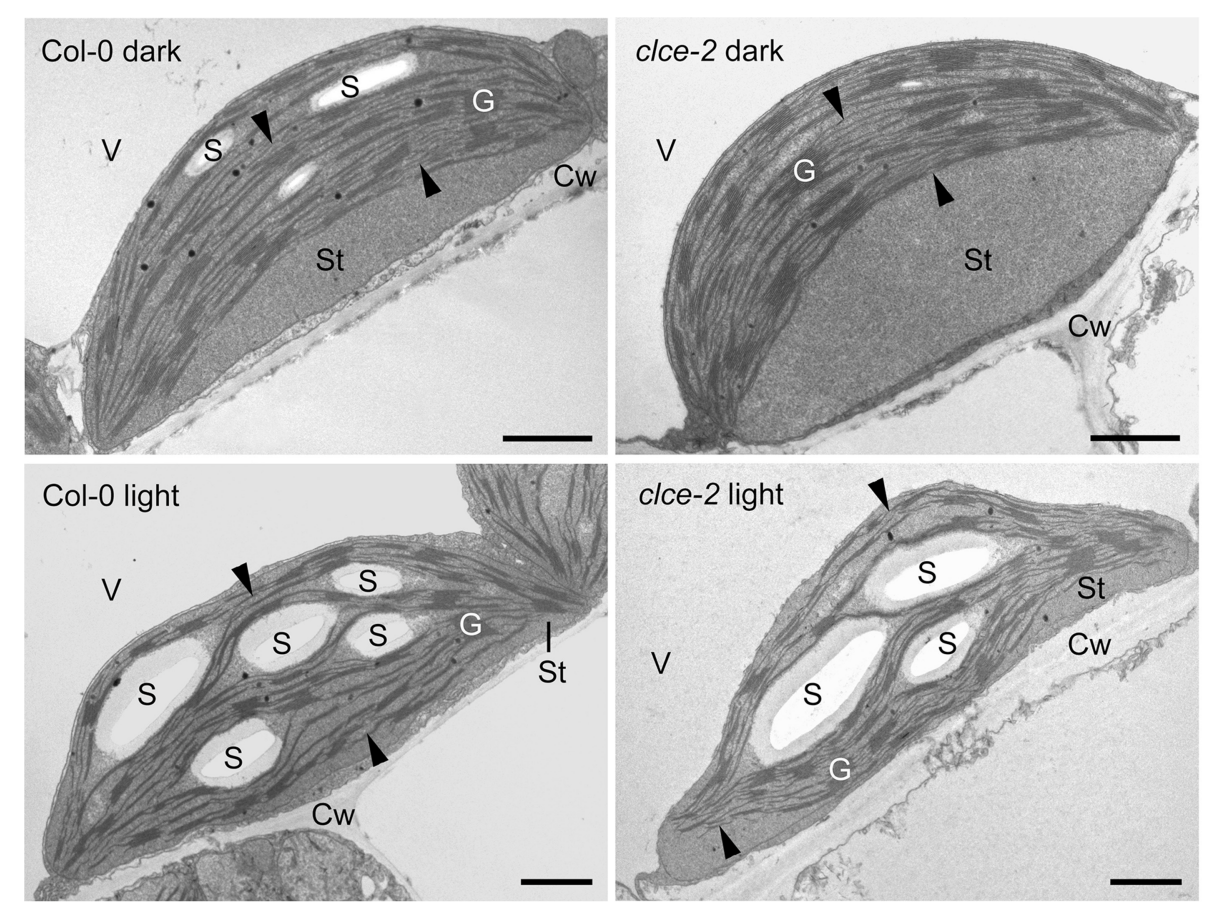

FIGURE 10 | Representative transmission electron micrographs of chloroplasts. Leaves from 7-week-old wild-type and mutant plants that were dark-adapted for $16 \mathrm{~h}$ ("dark") or further illuminated for $3 \mathrm{~h}$ at $120 \mu \mathrm{mol}$ photons $\mathrm{m}^{-2} \mathrm{~s}^{-1}$ ("light") were fixed in dim green light for electron microscopy. Arrowhead, stroma thylakoids; Cw, cell wall; G, granum; S, starch; St, chloroplast stroma; V, vacuole. Scale bar: $1 \mu \mathrm{m}$.

re-arrangements of the electron transport chain components in thylakoids.

\section{AtCLCe-a Cl- Channel or an $\mathrm{NO}_{3}^{-} / \mathrm{H}^{+}$ Exchanger?}

AtClCe has in general a modest homology with the other plant CLCs, and forms a distinct family branch together with AtCLCf (Barbier-Brygoo et al., 2011). Phylogenetic analyses indicated the presence of homologs in green algae, brown algae, diatoms and cyanobacteria, sharing 25-35\% identity with AtCLCe, but thus far none have been characterized (Pfeil et al., 2014).

CLCs were initially thought to be involved in $\mathrm{Cl}^{-}$transport after the first CLC was cloned, which was the voltage-dependent $\mathrm{Cl}^{-}$channel of torpedo fish (CLC-0; Jentsch et al., 1990). Crystal structures revealed bacterial CLCs as secondary active transporters that exchange $\mathrm{Cl}^{-}$and $\mathrm{H}^{+}$with a 2:1 stoichiometry (Accardi and Miller, 2004). Animal CLCs were found to function as $\mathrm{Cl}^{-}$channels at the plasma membrane or as $2 \mathrm{Cl}^{-} / 1 \mathrm{H}^{+}$exchangers in organellar membranes (Jentsch, 2015). Even though they catalyze distinct transport reactions, they share the basic protein architecture. More specifically, CLC proteins are homodimers with separate ion pathways within each monomer (Dutzler et al., 2002). Each monomer consists of a transmembrane component, which forms the ion transportation pathway, and in the case of eukaryotic members, also of a cytosolic cystathionine beta-synthase (CBS) domain component, which binds nucleotides and regulates the transmembrane component.
Multiple sequence alignment of AtCLCe with three CLCs from prokaryotes and eukaryotes indicated $17.9 \%$ identity with AtCLCa (De Angeli et al., 2006), 20.2\% with CLC from Cyanidioschyzon merolae (Feng et al., 2010), and 23.3\% with the CLCa from Escherichia coli (Dutzler et al., 2002; Supplementary Figure 2). AtCLCe sequence lacks many residues in the anion selectivity filter conserved in most CLCs (Dutzler et al., 2002; Feng et al., 2010). It does not harbor either the conserved serine in the $\mathrm{Cl}^{-}$binding site of crystallized CLCs or the proline residue, shown to be crucial for preference of $\mathrm{NO}_{3}^{-}$vs. $\mathrm{Cl}^{-}$in AtCLCa (Wege et al., 2010). Nevertheless, the positively charged residue (lysine) at this position may still be able to coordinate $\mathrm{Cl}^{-}$in the transportation pathway, but reduces the chances of a preference for transport of $\mathrm{NO}_{3}^{-}$. Thus the previous observation that clce mutants displayed altered nitrate accumulation (Monachello et al., 2009) could be an indirect effect due to possible alteration in the expression of $\mathrm{NO}_{3}^{-} / \mathrm{H}^{+}$exchangers from the AtCLC family. In addition, our data showing similar OJIP phenotype in untreated and $\mathrm{KNO}_{3}$-treated clce mutants (Figure 5) indicates that even if AtCLCe would play a role in nitrate homeostasis, this did not affect photosynthetic electron transport in thylakoids. Instead, AtCLCe appears to function in $\mathrm{Cl}^{-}$homeostasis, which affects electron transport via thylakoid re-arrangements.

AtCLCe sequence contains the so-called "gating glutamate" (Supplementary Figure 2), which is conserved in almost all CLCs and which protonation opens the $\mathrm{Cl}^{-}$transportation pathway (Feng et al., 2010). However, AtCLCe sequence does not contain the "proton glutamate" important for $\mathrm{H}^{+}$translocation, 
which could indicate that this CLC member is not an anion $\mathrm{H}^{+}$exchanger. Instead, at the "proton glutamate" position, a serine residue is present in AtCLCe and a threonine in algal CLC, which was shown to still function as an exchanger (Feng et al., 2010). In addition to serine and the gating glutamate, a tyrosine residue is involved in coordination of the $\mathrm{Cl}^{-}$ion in the translocation pathway. This residue is replaced by a threonine in AtCLCe, which is also polar and harbors a hydroxyl group important for coordination. Finally, the aspartate residue involved in ATP binding in the CBS domain (De Angeli et al., 2009) is replaced by a cysteine in AtCLCe. As reviewed by Pottosin and Dobrovinskaya (2015), electrophysiological studies of AtCLCe are required to determine the $\mathrm{Cl}^{-}$vs. $\mathrm{NO}_{3}^{-}$selectivity and channel vs. exchanger mechanism of transport for this protein. This will also allow to establish whether AtCLCe is responsible for the channel activity previously reported in thylakoids (Schönknecht et al., 1988).

\section{AUTHOR CONTRIBUTIONS}

$\mathrm{AH}, \mathrm{BL}$, and $\mathrm{CS}$ conceived the study and designed the experiments. AH carried out chlorophyll fluorescence, P700, ST,

\section{REFERENCES}

Accardi, A., and Miller, C. (2004). Secondary active transport mediated by a prokaryotic homologue of $\mathrm{ClC} \mathrm{Cl-} \mathrm{channels.} \mathrm{Nature} \mathrm{427,} \mathrm{803-807.} \mathrm{doi:}$ 10.1038 /nature 02314

Anderson, J. M., Horton, P., Kim, E. H., and Chow, W. S. (2012). Towards elucidation of dynamic structural changes of plant thylakoid architecture. Philos. Trans. R. Soc. Lond. B Biol. Sci. 367, 3515-3524. doi: 10.1098/rstb.2012.0373

Armbruster, U., Carrillo, L. R., Venema, K., Pavlovic, L., Schmidtmann, E., Kornfeld, A., et al. (2014). Ion antiport accelerates photosynthetic acclimation in fluctuating light environments. Nat. Commun. 5, 5439. doi: $10.1038 /$ ncomms6439

Barbier-Brygoo, H., De Angeli, A., Filleur, S., Frachisse, J. M., Gambale, F., Thomine, S., et al. (2011). Anion channels/transporters in plants: from molecular bases to regulatory networks. Annu. Rev. Plant Biol. 62, 25-51. doi: 10.1146/annurev-arplant-042110-103741

Barzda, V., Mustárdy, L., and Garab, G. (1994). Size dependency of circular dichroism in macroaggregates of photosynthetic pigment-protein complexes. Biochemistry 33, 10837-10841. doi: 10.1021/bi00201a034

Batelli, G., Verslues, P. E., Agius, F., Qiu, Q., Fujii, H., Pan, S., et al. (2007). SOS2 promotes salt tolerance in part by interacting with the vacuolar $\mathrm{H}^{+}$. ATPase and upregulating its transport activity. Mol. Cell Biol. 27, 7781-7790. doi: 10.1128/MCB.00430-07

Bellafiore, S., Barneche, F., Peltier, G., and Rochaix, J. D. (2005). State transitions and light adaptation require chloroplast thylakoid protein kinase STN7. Nature 433, 892-895. doi: 10.1038/nature03286

Carraretto, L., Formentin, E., Teardo, E., Checchetto, V., Tomizioli, M., Morosinotto, T., et al. (2013). A thylakoid-located two-pore $\mathrm{K}^{+}$channel controls photosynthetic light utilization in plants. Science 342, 114-118. doi: $10.1126 /$ science. 1242113

Chuartzman, S. G., Nevo, R., Shimoni, E., Charuvi, D., Kiss, V., Ohad, I., et al. (2008). Thylakoid membrane remodeling during state transitions in Arabidopsis. Plant Cell 20, 1029-1039. doi: 10.1105/tpc.107.055830

Cruz, J. A., Avenson, T. J., Kanazawa, A., Takizawa, K., Edwards, G. E., and Kramer, D. M. (2005). Plasticity in light reactions of photosynthesis for energy production and photoprotection. J. Exp. Bot. 56, 395-406. doi: $10.1093 /$ jxb/eri022 and ECS measurements. HN conducted screening of the mutants and RT-PCR. OZ carried out CD analyses. KS carried out TEM analyses. $\mathrm{AH}$ and $\mathrm{CS}$ wrote the manuscript. All authors helped to edit the manuscript.

\section{ACKNOWLEDGMENTS}

We are grateful to Csilla Jónás (Eötvös Loránd University) for skillful technical assistance in electron microscopic sample preparation. We thank to Giovanni Finazzi (CEA Grenoble) and Imre Vass (Biological Research Centre, Szeged) for useful discussions. This work was supported by the Swedish Research Council (CS), Carl Tryggers Foundation (BL), and Hungarian Research Fund OTKA K112688 (GG). HN was a recipient of a postdoctoral fellowship from Carl Tryggers Foundation.

\section{SUPPLEMENTARY MATERIAL}

The Supplementary Material for this article can be found online at: http://journal.frontiersin.org/article/10.3389/fpls.2016. 00115

Cruz, J. A., Sacksteder, C. A., Kanazawa, A., and Kramer, D. M. (2001). Contribution of electric field $(\Delta \psi)$ to steady-state transthylakoid proton motive force (pmf) in vitro and in vivo. Control of pmf parsing into $\Delta \psi$ and $\Delta \mathrm{pH}$ by ionic strength. Biochemistry 40, 1226-1237. doi: $10.1021 /$ bi00 18741

Cseh, Z., Rajagopal, S., Tsonev, T., Busheva, M., Papp, E., and Garab, G. (2000). Thermooptic effect in chloroplast thylakoid membranes. Thermal and light stability of pigment arrays with different levels of structural complexity. Biochemistry 39, 15250-15257. doi: 10.1021/bi001600d

Damkjaer, J. T., Kereïche, S., Johnson, M. P., Kovacs, L., Kiss, A. Z., Boekema, E. J., et al. (2009). The photosystem II light-harvesting protein Lhcb3 affects the macrostructure of photosystem II and the rate of state transitions in Arabidopsis. Plant Cell 21, 3245-3256. doi: 10.1105/tpc.108.064006

De Angeli, A., Monachello, D., Ephritikhine, G., Frachisse, J. M., Thomine, S., Gambale, F., et al. (2006). The nitrate/proton antiporter AtCLCa mediates nitrate accumulation in plant vacuoles. Nature 442, 939-942. doi: 10.1038 /nature 05013

De Angeli, A., Moran, O., Wege, S., Filleur, S., Ephritikhine, G., Thomine, S., et al. (2009). ATP binding to the C terminus of the Arabidopsis thaliana nitrate/proton antiporter, AtCLCa, regulates nitrate transport into plant vacuoles. J. Biol. Chem. 284, 26526-26532. doi: 10.1074/jbc.M109. 005132

De Angeli, A., Thomine, S., Frachisse, J. M., Ephritikhine, G., Gambale, F., and Barbier-Brygoo, H. (2007). Anion channels and transporters in plant cell membranes. FEBS Lett. 581, 2367-2374. doi: 10.1016/j.febslet.2007. 04.003

Dutzler, R., Campbell, E. B., Cadene, M., Chait, B. T., and MacKinnon, R. (2002). $\mathrm{X}$-ray structure of a $\mathrm{ClC}$ chloride channel at 3.0 A reveals the molecular basis of anion selectivity. Nature 415, 287-294. doi: 10.1038/415287a

Feng, L., Campbell, E. B., Hsiung, Y., and MacKinnon, R. (2010). Structure of a eukaryotic CLC transporter defines an intermediate state in the transport cycle. Science 330, 635-641. doi: 10.1126/science.1195230

Finazzi, G., Petroutsos, D., Tomizioli, M., Flori, S., Sautron, E., Villanova, V., et al. (2015). Ions channels/transporters and chloroplast regulation. Cell Calcium 58, 86-97. doi: 10.1016/j.ceca.2014.10.002

Garab, G., Kieleczawa, J., Sutherland, J. C., Bustamante, C., and Hind, C. (1991). Organization of pigment-protein complexes into macrodomains in the thylakoid membranes of wild-type and chlorophyll b-less mutant of barley 
as revealed by circular dichroism. Photochem. Photobiol. 54, 273-281. doi: 10.1111/j.1751-1097.1991.tb02016.x

Garab, G., and van Amerongen, H. (2009). Linear dichroism and circular dichroism in photosynthesis research. Photosynth. Res. 101, 135-146. doi: 10.1007/s11120-009-9424-4

Guo, W., Zuo, Z., Cheng, X., Sun, J., Li, H., Li, L., et al. (2014). The chloride channel family gene CLCd negatively regulates pathogen-associated molecular pattern (PAMP)-triggered immunity in Arabidopsis. J. Exp. Bot. 65, 1205-1215. doi: $10.1093 / \mathrm{jxb} / \mathrm{ert} 484$

Guskov, A., Kern, J., Gabdulkhakov, A., Broser, M., Zouni, A., and Saenger, W. (2009). Cyanobacterial photosystem II at 2.9- $\AA$ resolution and the role of quinones, lipids, channels and chloride. Nat. Struct. Mol. Biol. 16, 334-342. doi: 10.1038/nsmb.1559

Hind, G., Nakatani, H. Y., and Izawa, S. (1974). Light-dependent redistribution of ions in suspensions of chloroplast thylakoid membranes. Proc. Natl Acad. Sci. U.S.A. 71, 1484-1488. doi: 10.1073/pnas.71.4.1484

Jentsch, T. J. (2015). Discovery of CLC transport proteins: cloning, structure, function and pathophysiology. J. Physiol. 593, 4091-4109. doi: 10.1113/ jp270043

Jentsch, T. J., Steinmeyer, K., and Schwarz, G. (1990). Primary structure of Torpedo marmorata chloride channel isolated by expression cloning in Xenopus oocytes. Nature 348, 510-514. doi: 10.1038/348510a0

Jossier, M., Kroniewicz, L., Dalmas, F., Le Thiec, D., Ephritikhine, G., Thomine, S., et al. (2010). The Arabidopsis vacuolar anion transporter, AtCLCc, is involved in the regulation of stomatal movements and contributes to salt tolerance. Plant J. 64, 563-576. doi: 10.1111/j.1365-313X.2010.04352.x

Kirchhoff, H. (2013). Architectural switches in plant thylakoid membranes. Photosynth. Res. 116, 481-487. doi: 10.1007/s11120-013-9843-0

Kirchhoff, H., Hall, C., Wood, M., Herbstová, M., Tsabari, O., Nevo, R., et al. (2011). Dynamic control of protein diffusion within the granal thylakoid lumen. Proc. Natl. Acad. Sci. U.S.A. 108, 20248-20253. doi: 10.1073/pnas.1104141109

Kramer, D. M., Cruz, J. A., and Kanazawa, A. (2003). Balancing the central roles of the thylakoid proton gradient. Trends Plant Sci. 8, 27-32. doi: 10.1016/S13601385(02)00010-9

Krapp, A., David, L. C., Chardin, C., Girin, T., Marmagne, A., Leprince, A. S., et al. (2014). Nitrate transport and signalling in Arabidopsis. J. Exp. Bot. 65, 789-798. doi: $10.1093 / \mathrm{jxb} / \mathrm{eru} 001$

Kunz, H. H., Gierth, M., Herdean, A., Satoh-Cruz, M., Kramer, D. M., Spetea, C., et al. (2014). Plastidial transporters KEA1, -2 , and -3 are essential for chloroplast osmoregulation, integrity, and $\mathrm{pH}$ regulation in Arabidopsis. Proc. Natl. Acad. Sci. U.S.A. 111, 7480-7485. doi: 10.1073/pnas.1323899111

Lichtenthaler, H. K., and Wellburn, A. R. (1983). Determinations of total carotenoids and chlorophylls $a$ and $b$ of leaf extracts in different solvents. Biochem. Soc. Trans. B 603, 591-592. doi: 10.1042/bst0110591

Lunde, C., Jensen, P. E., Haldrup, A., Knoetzel, J., and Scheller, H. V. (2000). The PSI-H subunit of photosystem I is essential for state transitions in plant photosynthesis. Nature 408, 613-615. doi: 10.1038/350 46121

Lv, Q. D., Tang, R. J., Liu, H., Gao, X. S., Li, Y. Z., Zheng, H. Q., et al. (2009). Cloning and molecular analyses of the Arabidopsis thaliana chloride channel gene family. Plant Sci. 176, 650-661. doi: 10.1016/j.plantsci.2009. 02.006

Marmagne, A., Vinauger-Douard, M., Monachello, D., De Longevialle, A. F., Charon, C., Allot, M., et al. (2007). Two members of the Arabidopsis CLC (chloride channel) family, AtCLCe and AtCLCf, are associated with thylakoid and Golgi membranes, respectively. J. Exp. Bot. 58, 3385-3393. doi: 10.1093/jxb/erm187

Monachello, D., Allot, M., Oliva, S., Krapp, A., Daniel-Vedele, F., BarbierBrygoo, H., et al. (2009). Two anion transporters AtClCa and AtClCe fulfil interconnecting but not redundant roles in nitrate assimilation pathways. New Phytol. 183, 88-94. doi: 10.1111/j.1469-8137.2009.02837.x

Nash, D., Miyao, M., and Murata, N. (1985). Heat inactivation of oxygen evolution in photosystem II particles and its acceleration by chloride depletion and exogenous manganese. Biochim. Biophys. Acta 807, 127-133. doi: 10.1016/00052728(85)90115-X

Nguyen, C. T., Agorio, A., Jossier, M., Depré, S., Thomine, S., and Filleur, S. (2015). Characterization of the chloride channel-like, AtCLCg, involved in chloride tolerance in Arabidopsis thaliana. Plant Cell Physiol. doi: 10.1093/pcp/pcv169. [Epub ahead of print].

Nilkens, M., Kress, E., Lambrev, P., Miloslavina, Y., Müller, M., Holzwarth, A. R., et al. (2010). Identification of a slowly inducible zeaxanthin-dependent component of non-photochemical quenching of chlorophyll fluorescence generated under steady-state conditions in Arabidopsis. Biochim. Biophys. Acta 1797, 466-475. doi: 10.1016/j.bbabio.2010.01.001

Pfeil, B. E., Schoefs, B., and Spetea, C. (2014). Function and evolution of channels and transporters in photosynthetic membranes. Cell Mol. Life Sci. 71, 979-998. doi: $10.1007 / \mathrm{s} 00018-013-1412-3$

Pottosin, I., and Dobrovinskaya, O. (2015). Ion channels in native chloroplast membranes: challenges and potential for direct patch-clamp studies. Front. Physiol. 6:396. doi: 10.3389/fphys.2015.00396

Pottosin, I. I., and Schönknecht, G. (1995). Patch clamp study of the voltagedependent anion channel in the thylakoid membrane. J. Membr. Biol. 148, 143-156. doi: 10.1007/BF00207270

Pottosin, I., and Shabala, S. (2015). Transport across chloroplast membranes: optimizing photosynthesis for adverse environmental conditions. Mol. Plant. doi: 10.1016/j.molp.2015.10.006. [Epub ahead of print].

Pribil, M., Labs, M., and Leister, D. (2014). Structure and dynamics of thylakoids in land plants. J. Exp. Bot. 65, 1955-1972. doi: 10.1093/jxb/ eru090

Ruban, A. V., Johnson, M. P., and Duffy, C. D. (2012). The photoprotective molecular switch in the photosystem II antenna. Biochim. Biophys. Acta 1817, 167-181. doi: 10.1016/j.bbabio.2011.04.007

Schansker, G., Tóth, S. Z., and Strasser, R. J. (2005). Methylviologen and dibromothymoquinone treatments of pea leaves reveal the role of photosystem I in the Chl a fluorescence rise OJIP. Biochim. Biophys. Acta 1706, 250-261. doi: 10.1016/j.bbabio.2004.11.006

Schönknecht, G., Hedrich, R., Junge, W., and Raschke, K. (1988). A voltagedependent chloride channel in the photosynthetic membrane of a higher-plant. Nature 336, 589-592. doi: 10.1038/336589a0

Schreiber, U., and Klughammer, C. (2008). New accessory for the DUAL-PAM100: The P515/535 module and examples of its application. PAM Appl. Notes 1, 1-10. Available online at: http://walz.com/downloads/pan/PAN07001_ed2.pdf

Solymosi, K., and Bertrand, M. (2012). Soil metals, chloroplasts, and secure crop production: a review. Agron. Sustain. Dev. 32, 245-272. doi: 10.1007/s13593011-0019-z

Strasser, R. J., Srivastava, A., and Tsimilli-Michael, M. (2000). "The fluorescence transient as a tool to characterize and screen photosynthetic samples," in Probing Photosynthesis: Mechanism, Regulation and Adaptation, eds M. Yunus, U. Pathre, and P. Mohanty (London: Taylor and Francis), $445-483$.

Strasser, R. J., Tsimilli-Michael, M., and Srivastava, A. (2004). "Analysis of the chlorophyll a fluorescence transient," in Chlorophyll a Fluorescence: A Signature of Photosynthesis, eds G. C. Papageorgiou and Govindjee (Dordrecht: SpringerVerlag), 321-362.

Takizawa, K., Kanazawa, A., and Kramer, D. M. (2008). Depletion of stromal $\mathrm{Pi}$ induces high 'energy-dependent'antenna exciton quenching ( $\mathrm{qE}$ ) by decreasing proton conductivity at $\mathrm{CFO}-\mathrm{CF} 1 \mathrm{ATP}$ synthase. Plant Cell Environ. 31, 235-243. doi: 10.1111/j.1365-3040.2007.01753.x

Teardo, E., Frare, E., Segalla, A., De Marco, V., Giacometti, G. M., and Szabò, I. (2005). Localization of a putative ClC chloride channel in spinach chloroplasts. FEBS Lett. 579, 4991-4996. doi: 10.1016/j.febslet.2005. 08.005

Tikkanen, M., Piippo, M., Suorsa, M., Sirpiö, S., Mulo, P., Vainonen, J., et al. (2006). State transitions revisited-a buffering system for dynamic low light acclimation of Arabidopsis. Plant Mol. Biol. 62, 779-793. doi: 10.1007/s11103-0069088-9

Umena, Y., Kawakami, K., Shen, J.-R., and Kamiya, N. (2011). Crystal structure of oxygen-evolving photosystem II at a resolution of $1.9 \AA$ A Nature $473,55-60$. doi: 10.1038/nature 09913

von der Fecht-Bartenbach, J., Bogner, M., Dynowski, M., and Ludewig, U. (2010). CLC-b-mediated NO-3/H+ exchange across the tonoplast of Arabidopsis vacuoles. Plant Cell Physiol. 51, 960-968. doi: 10.1093/pcp/ pcq062

von der Fecht-Bartenbach, J., Bogner, M., Krebs, M., Stierhof, Y. D., Schumacher, K., and Ludewig, U. (2007). Function of the anion transporter AtCLC-d 
in the trans-Golgi network. Plant J. 50, 466-474. doi: 10.1111/j.1365313X.2007.03061.x

Wege, S., Jossier, M., Filleur, S., Thomine, S., Barbier-Brygoo, H., Gambale, F., et al. (2010). The proline 160 in the selectivity filter of the Arabidopsis $\mathrm{NO}(3)(-) / \mathrm{H}(+)$ exchanger AtCLCa is essential for nitrate accumulation in planta. Plant J. 63, 861-869. doi: 10.1111/j.1365-313X.2010.04288.x

Yoshioka-Nishimura, M., Nanba, D., Takaki, T., Ohba, C., Tsumura, N., Morita, N., et al. (2014). Quality control of photosystem II: direct imaging of the changes in the thylakoid structure and distribution of $\mathrm{FtsH}$ proteases in spinach chloroplasts under light stress. Plant Cell Physiol. 55, 1255-1265. doi: $10.1093 / \mathrm{pcp} / \mathrm{pcu} 079$
Conflict of Interest Statement: The authors declare that the research was conducted in the absence of any commercial or financial relationships that could be construed as a potential conflict of interest.

Copyright (C) 2016 Herdean, Nziengui, Zsiros, Solymosi, Garab, Lundin and Spetea. This is an open-access article distributed under the terms of the Creative Commons Attribution License (CC BY). The use, distribution or reproduction in other forums is permitted, provided the original author(s) or licensor are credited and that the original publication in this journal is cited, in accordance with accepted academic practice. No use, distribution or reproduction is permitted which does not comply with these terms. 